\title{
Research in Space Science
}

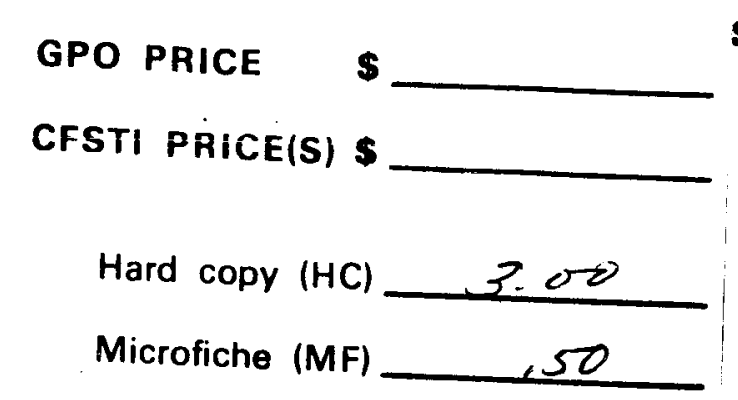

\# 653 July 65

SPECIAL REPORT

Number 219

METEOR MASSES AND LUMINOSITY

Franco Verniani

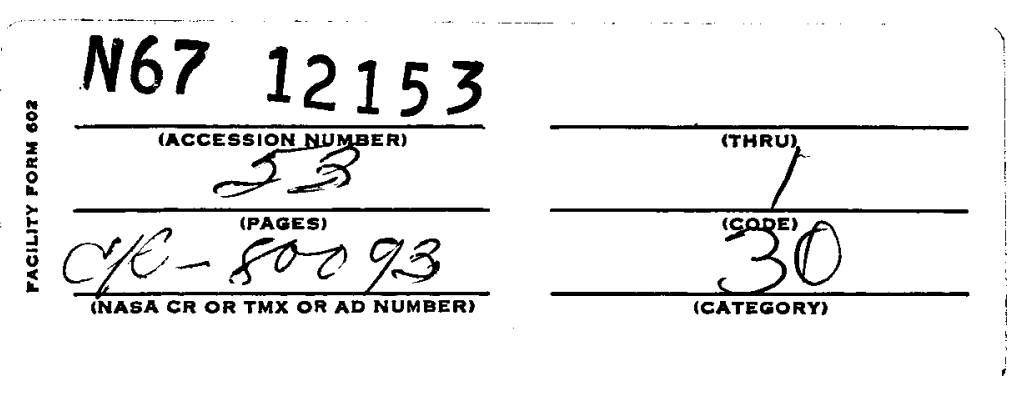

August 26, 1966

1 
SAO Special Report No. 219

METEOR MASSES $\triangle N D$ LUMINOSITY

Franco Verniani

Smithsonian Institution Astrophysical Observatory Cambridge, Massachusetts 02138 
TABLE OF CONTENTS

$\underline{\text { Section }}$

$\underline{\text { Page }}$

ABSTRACT ................... ix

1. INTRODUCTION $\ldots \ldots \ldots \ldots \ldots \ldots \ldots \ldots \ldots$

2. THE VELOCITY DEPENDENCE OF THE LUMINOUS

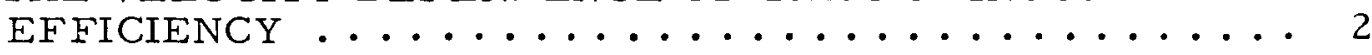

3. THE LUMINOUS EFFICIENCY COEFFICIENT T op $\ldots \ldots . . . \ldots 22$

4. METEOR DENSITIES ...................... 32

5. REFERENCES .......................... 44 
Table

Page

1. Mean values of $\left(\log T_{\mathrm{p}} / \rho_{\mathrm{m}}^{2}\right) \mathrm{s}=0$ and other physical characteristics as a function of velocity $v$ for 189 sporadic Super-Schmidt meteors . . . . . . . . . 9

2. Mean values of $\left(\log \tau_{\mathrm{p}} / \rho_{\mathrm{m}}^{2}\right)_{\mathrm{s}}=0$ and other physical characteristics as a function of the aphelion distance $Q$ for 189 sporadic Super-Schmidt meteors ........ 10

3. Mean values $\left(\log \tau_{\mathrm{p}} / \rho_{\mathrm{m}}^{2}\right)_{\mathrm{s}}=0$ as a function of velocity, reduced to the density $\rho_{\mathrm{m}}^{\prime}$ of the long-period meteors . . 12

4. Mean values of $\left(\log \tau_{\mathrm{p}} / \rho_{\mathrm{m}}^{2}\right)_{\mathrm{s}}=0$ as a function of the brightness $\epsilon_{\infty}$ for 189 sporadic Super-Schmidt meteors . 15

5. Mean values of $\left(\log \tau_{\mathrm{p}} / \mathrm{p}_{\mathrm{m}}^{2}\right)_{\mathrm{s}}=0$ as a function of the mass $m_{\infty}$ for 189 sporadic Super-Schmidt meteors ... 17

6. Mean values of $\left(\log \tau_{p} / p_{m}^{2}\right)_{s}=0$ as a function of the fragmentation index $\chi$ for 189 sporadic Super-Schmidt

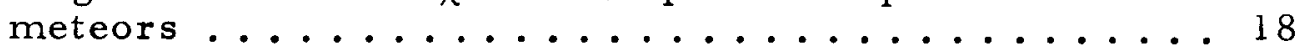

7. Mean values of $\left(\tau_{\mathrm{p}} / \rho_{\mathrm{m}}^{2}\right)_{\mathrm{s}}=0$ as a function of the ablation coefficient $\sigma$ for 189 sporadic Super-Schmidt

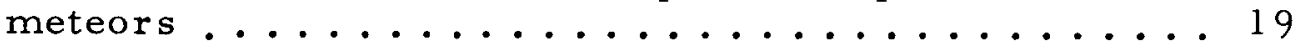

8. The mean logarithmic density as a function of the aphelion distance, Q, for 220 sporadic Super-Schmidt

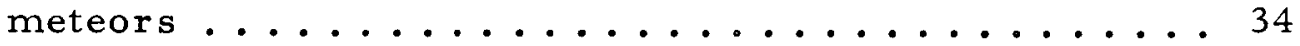

9. Average densities for Super-Schmidt shower

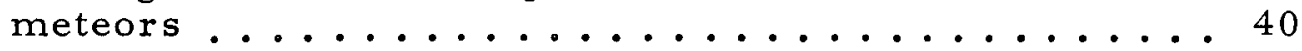

10. Summary of the densities of 322 Super-Schmidt meteors . 43 


\section{LIST OF ILIUSTRATIONS}

Figure

Page

1. Mean values of $\left(\log T_{p} / \rho_{\mathrm{m}}^{2}\right) \mathrm{s}=0$, reduced to the density of long-period meteors, as functions of velocity for 189 sporadic Super-Schmidt meteors....

2. Mean values of $\left(\log \tau \mathrm{p} / \rho_{\mathrm{m}}^{2}\right)_{\mathrm{s}}=0$, reduced to constant velocity and to the density of long-period meteors, as functions of the ablation coefficient $\sigma$ for 189 sporadic Super-Schmidt nictcors.........

3. The decimal logarithm of the observed-to-theoretical deceleration ratio, a/a $\mathrm{T}$, plotted versus the mass-loss parameter s for Meteor No. 7946 ......

4. The decimal logarithm of the observed-to-theoretical deceleration ratio, a/a $\mathrm{T}$, plotted versus the mass-loss parameter s for Meteor No. $19816 \ldots 26$

5. The decimal logarithm of the observed-to-theoretical deceleration ratio, a/a $\mathrm{T}$, plotted versus the mass-loss paramater s for Meteor No. $1242 \ldots . . . .28$

6. The density distribution for 220 sporadic Super-

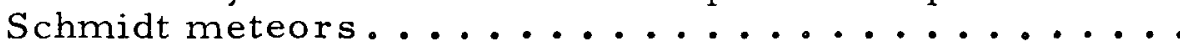

7. The density of 220 sporadic Super-Schmidt meteors, as function of the a phelion distance $Q \ldots \ldots \ldots$

8. The density distribution for two groups of sporadic Super-Schmidt meteors, respectively, in short-

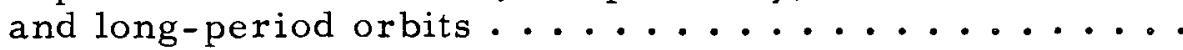

9. The density distribution for three subgroups of

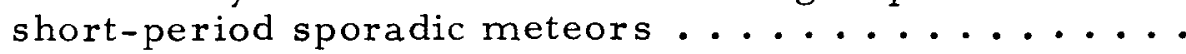

10. The density distribution for three meteor showers: The Geminids, a Capricornids, and Southern Taurids . . 


\title{
PrEeEDING PAGE BLANK NOT FILMED.
}

\begin{abstract}
In 1964 the author published a study of the meteor luminous efficiency based on Harvard's photographic data. This paper presents a more sophisticated analysis of the same data since it takes better into account the effects of fragmentation. On the whole, 1964 results are confirmed, but the accuracy is greatly improved. The luminous effiriency $\tau_{p}$ can be expressed as a function of velocity simply as $\tau_{p}=1.0 \times 10^{-19} \mathrm{v}$, with $\mathrm{v}$ in $\mathrm{cm} \mathrm{sec}^{-1}$ and the luminosity expressed in units of 0 -mag stars. The uncertainty is \pm 0.1 in the velocity exponent and about 40 percent in the coefficient. It is confirmed that, in the mass range $10^{-2}-30 \mathrm{~g}$, the luminous efficiency does not depend eithe $r$ on mass or on brightness, nor does it show any appreciable variation in the course of the trajectory. The coefficient, $\tau_{\text {op }}=1 \times 10^{-19}$ 0 mag $\mathrm{g}^{-1} \mathrm{~cm}^{-3} \mathrm{sec}^{4}$, has been established by means of the data on three asteroidal meteors. Once the mass scale has been established, meteor densities have been determined. Most meteors, both sporadic and shower, have an average density in the vicinity of $0.2 \mathrm{~g} \mathrm{~cm}^{-3}$. It appears, however, that a small group of sporadic meteors in very short orbits exists, with higher densities of the order of $1 \mathrm{~g} \mathrm{~cm}^{-3}$. Because of selection effects, it is impossible to estimate the actual consistency of this group; it is clear, however, that it must represent a very small minority of cometary meteors.
\end{abstract}




\title{
METEOR MASSES AND LUMINOSITY ${ }^{l}$
}

\author{
Franco Verniani ${ }^{2}$
}

\section{INTRODUCTION}

A comprehensive study of the luminous efficiency of meteors was made by the author (Verniani, 1964a, hereafter referred to as Paper I) using the photographic material gathered over many years under the Harvard Photographic Meteor Project. Assuming, as was customary, a dependence of the luminous efficiency $\tau_{p}$ on the meteor velocity $v$ of the form $\tau_{p}=\tau_{o p} v^{n}$, the author found $n=1.0$ with a probable error of \pm 0.15 , and $\tau_{\text {op }}=1 \times 10^{-19} 0 \mathrm{mag} \mathrm{g}^{-1} \mathrm{~cm}^{-3} \mathrm{sec}^{4}$ with an uncertainty of the order of a factor of 2 or more. The results described in Paper I showed no dependence of the photographic luminous efficiency $T_{p}$ either on the mass or on the brightness of meteors, and no appreciable systematic change of $T_{p}$ in the course of the meteor trajectories.

At the time that this study was carried out, in 1963, the general analysis of the atmospheric trajectories of the precisely reduced meteors was in its early stages. By the time the general analysis was completed by Jacchia, Verniani, and Briggs (1965), the knowledge acquired suggested the possibility of improving the accuracy of the luminous efficiency, both in its coefficient $T_{o p}$ and in its exponent $n$. The new determinations of $\tau_{\text {op }}$ and $n$ will be discussed separately in the following sections.

\footnotetext{
This work was supported in part by Grant NSR-09-015-033 from the National Aeronautics and Space Administration.

${ }^{2}$ Physicist, on leave from Centro Nazionale per la Fisica dell'Atmosfera e la Meteorologia del C. N. R., Rome, Italy.
} 


\section{THE VELOCITY DEPENDENCE OF THE LUMINOUS EFFICIENCY}

The photographic luminous efficiency $\tau_{\mathrm{p}}$ is defined, as in Paper I, by the so-called luminosity equation:

$$
I_{p}=-\frac{1}{2} T_{p} v^{2} \frac{d m}{d t}
$$

where $I_{p}$ is the luminous flux ${ }^{2}$ in the wavelength range of the blue plate (roughly 0.38 to $0.55 \mu$ ), and $m$ is the meteor mass.

The drag equation, expressing the conservation of momentum in the interactions of the meteoroid with the molecules of the atmosphere, is

$$
a=\gamma A \rho_{m}^{-2 / 3} m^{-1 / 3} \rho_{a} v^{2}
$$

where a and $\rho_{\mathrm{m}}$ are the deceleration and the density of the meteor, respectively, $\gamma$ is the drag coefficient, $A$ is the shape factor, and $\rho_{a}$ is the atmospheric density. By integrating the luminosity equation and combining the result with the drag equation, we obtain

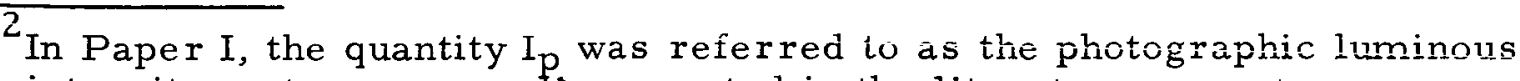
intensity, a term generally accepted in the literature on meteors. The new name-flux instead of intensity-is in agreement with the recommendations of the Commission for Symbols, Units, and Nomenclature of the International Union of Pure and Applied Physics (Wolfe, 1962).
} 


$$
\frac{{ }^{\tau} \mathrm{p}}{\rho_{\mathrm{m}}^{2}}=\frac{2 \mathrm{E}}{\bar{v}^{2}}\left(\frac{\mathrm{a}}{\gamma A \rho_{\mathrm{a}} v^{2}}\right)^{3},
$$

where

$$
E=\int_{t}^{t} I_{p} d t
$$

is the integrated brightness, $\vec{v}$ is a value of $v$ at some instant between $t$ and $t_{E}$, and $t_{E}$ is the instant at which the meteor luminosity ceases to be detectable on the photographic plate. The observational data allow the determination of the velocity $v$, deceleration $a$, integrated brightness $E$, and height $z$; the U.S. Standard Atmosphere 1962 (1962) gives $\rho_{a}$ as a function of $z$. For a given meteor, $\bar{v}$ varies only slightly with $t$. It was shown in Paper I that the velocity at $m=m_{\infty} / 2$ represents an adequate approximation of $\bar{v}$. Most of the meteors photographed with the Super-Schmidt cameras have probably experienced free-molecule flow (see Paper I and Jacchia, Verniani, and Briggs, 1965), and therefore justify the use of the constant value 1.1 for $\gamma$. The shape factor is unknown. In Paper $I$ we adopted $A=1.21$, the value for a sphere, as used in all earlier Harvard works. For any shape other than spherical and with random orientation, A will have a somewhat larger value. Here, following Jacchia, Verniani, and Briggs, (1965), we shall assume $A=1.5$.

Equation (3) allows us to compute $\tau_{p} / \rho_{\text {II }}^{2}$ from the observational data. This equation, however, is valid only when the "dynamic" mass appearing in the drag equation is equal to the "photometric" mass obtained from the integration of the light curve. When fragmentation 
occurs, the values of $T_{p} / \rho_{m}^{2}$ computed from equation (3) require a correction, since the dynamic mass becomes smaller than the photometric mass. It was shown in Paper I that the relation between the values of $\tau_{\mathrm{p}} / \rho_{\mathrm{m}}^{2}$ computed at two different points of the trajectory is

$$
\left(\log \tau_{p} / \rho_{m}^{2}\right)_{s}=\left(\log \tau_{p} / \rho_{m}^{2}\right)_{s_{0}}+3 x\left(s-s_{0}\right),
$$

where $x$ is the fragmentation index introduced by Jacchia (1955) and defined by

$$
x=\frac{d}{d s} \log \frac{a}{a_{T}},
$$

a being the observed deceleration, $a_{T}$ the deceleration computed from the drag equation and the photometric mass, and $s$ the mass-loss parameter $s=\log \left[\left(\mathrm{m}_{\infty} / \mathrm{m}\right)-1\right]$. Let us consider a meteor for which it was possible to determine several decelerations at different points of its trajectory. The difference between the values of $s$ corresponding to the first and the last deceleration is of the order of 1 . An average value of $x$ is 0.25 . Consequently, for a typical long meteor, the fragmentation causes the value of $\tau_{p} / \rho_{m}^{2}$ computed from equation (3) with the last deceleration to be larger by a factor of 6 than the value computed with the first deceleration. Equation (4) would allow us to reduce all the values of $\tau_{p} / \rho_{\text {ril }}^{2}$ computed from equation (3) to the point of beginning of fragmentation, if only we could know where this point is for each meteor. Since, generally, this point is not known, no correction was applied in Paper $I$ to the values of ${ }^{\mathrm{p}} \mathrm{p} / \rho_{\mathrm{m}}^{2}$ computed 
from equation (3). Although it was shown empirically in Paper I that the system of weights ${ }^{3}$ employed in the analysis greatly reduced the effects of fragmentation and allowed a correct determination of the exponent $\mathrm{n}$, it is clear that the use of a direct correction would improve the accuracy of the determination of $n$.

In the general analysis of Jacchia, Verniani, and Briggs (1965), the meteor decelerations were used to derive atmospheric densities in order to study their seasonal fluctuations. The atmospheric densities, computed from the drag equation and the photometric masses,

${ }^{3}$ Velocities and heights are quite accurate, even in the worst cases. Decelerations, on the contrary, are not so well determined and their accuracy varies from meteor to meteor (see Jacchia, Verniani, and Briggs, 1965). As a consequence, in the determination of $\tau_{\mathrm{p}} / \rho_{\mathrm{m}}^{2}$ for each meteor, a weight $\mathrm{p}_{i}$ was given to each deceleration pertaining to the meteor. The weight $\mathrm{p}_{i}$ is defined by

$$
p_{i}=10 \psi\left(\frac{a_{i}}{p \cdot e \cdot{ }_{i}}\right) \cdot \psi\left(n_{i}-3\right) \cdot\left(\frac{m_{i}}{m_{\infty}}\right)^{1 / 2},
$$

where p.e. ${ }_{i}$ is the probable error of the deceleration $a_{i}, n_{i}$ is the number of segments used in the computation of $a_{i}$, and $\psi(x)$ is defined by

$$
\psi(x)=\frac{1}{2}[1+\operatorname{erf}(2.5 \log x-2.1)] .
$$

The factor $\left(m_{i} / m_{\infty}\right)^{1 / 2}$ is introduced to decrease the importance of decelerations near the end of trajectories, where fragmentation might be so severe as to make a correction meaningless. In our analysis we have assigned a weight $w=\left(\sum_{i} p_{i}\right)^{1 / 2}$ to the value of $\tau_{p} / p_{m}^{2}$ for each meteor. A thorough discussion on this system of weights is given in Paper I. 
had to be corrected for the fragmentation effects by reducing them to a given point in the trajectory. Using the least-squares method, Jacchia, Verniani, and Briggs (1965) showed that the scatter in the differences between the atmospheric densities computed from meteor decelerations and the densities given by the U. S. Standard Atmosphere 1962 (1962) was minimized for $s_{0}=0$. It is clear that the same value $s_{0}=0$ applies for the correction of the values of ${ }^{T} \rho_{p} / \rho_{m}^{2}$. We have then

$$
\left(\log \tau_{p} / \rho_{m}^{2}\right)_{s=0}=\left(\log \tau_{p} / \rho_{m}^{2}\right)_{s}-3 \times s
$$

In the analysis described in this section, values of $\left(\log \tau_{\mathrm{p}} / \mathrm{p}_{\mathrm{m}}^{2}\right)$ reduced to $s=0$ are used throughout.

Of the 413 original precisely reduced Super-Schmidt meteors, 12 did not allow the computation of the fragmentation index. We were left with 401 meteors, 281 sporadic and 120 shower meteors. Since each shower has its own peculiar density $\rho_{m}$, the sporadic meteors only can be used in the determination of $n$. In Paper I, 33 sporadic meteors having clearly anomalous values of $\tau_{p} / \rho_{m}^{2}$ were eliminated from the analysis. Some of those meteors showed either an abrupt beginning or a very short, flare-like light curve. For both these kinds of meteors, the use of the fragmentation index is generally not sufficient to correct the value of $\tau_{p} / \rho_{m}^{2}$ (see Paper I). In the present analysis we have eliminated from the sporadic sample all abrupt-beginning meteors (19), all flare-like meteors (10), all meteors with $x \geq 0.7$ (25), all meteors with $\log \sigma \geq-10.6$ (4) or without any computed value of $\sigma(1)$ (the ablation coefficient $\sigma$ is defined by the mass equation $\sigma=\dot{m} / \underline{m} v \dot{v}$ ), the asteroidal Meteor No. 7946, and a meteor having $w<1$. We were left with 220 sporadic meteors. 
The values of $\left(\log \tau_{p} / p_{m}^{2}\right)_{s}=0$ range, in cgs units, from -9.68 to -13.55 ; the weighted average is -11.44 and the standard deviation of 1 observation is \pm 0.80 . Since the scatter is so large, it is clear that we can reach a bette $r$ accuracy in the determination of $n$ if we use only the best part of the data. Some of the scatter, due to the differences in velocity, may be removed by the provisional as sumption that $\mathrm{n}=1$ in order to compute the values of $\left(\log \tau_{\text {op }} / \rho_{\mathrm{m}}^{2}\right)_{\mathrm{s}}=0^{.}$These quantities range from -16.52 to -20.04 ; the average is -17.88 and the standard deviation of 1 observation is \pm 0.75 . We will discard all those meteors whose values of $\left(\log \tau_{\text {op }} / \rho_{\mathrm{m}}^{2}\right)_{\mathrm{s}}=0$ differ from the average by more than 1. 5 times the standard deviation of 1 observation; i. e., more than \pm 1.12 . We are thus left with 189 meteors whose values of $\left(\log T_{\mathrm{op}} / \rho_{\mathrm{m}}^{2}\right)_{\mathrm{s}}=0$ range from -16.78 to -19.01 . In this manner, by eliminating only 31 meteors, or less than 15 percent of the material, we considerably reduce the scatter, from a total range of 3.52 to one of 2.23. Also the standard deviation of 1 observation is reduced, from \pm 0.75 to \pm 0.52 . As far as the $\left(\log \tau_{\mathrm{p}} / \rho_{\mathrm{m}}^{2}\right)_{\mathrm{s}}=0$ values are concerned, they range now from -10.04 to -12.84 , where the standard deviation of 1 observation is \pm 0.56 .

Before proceeding to the determination of $n$, we shall consider in some detail the causes of the large scatter found in the values of $\left(\log \tau_{\mathrm{p}} / \rho_{\mathrm{m}}^{2}\right)_{\mathrm{s}}=0$. The error $\Delta$ in one individual value of $\left(\log \tau_{\mathrm{p}} / \rho_{\mathrm{m}}^{2}\right)_{\mathrm{s}}=0$ can be easily expressed as a function of the uncertainties in the quantities used for its determination, as:

$$
\begin{aligned}
\Delta & =\left\{\left(\frac{\Delta \mathrm{E}}{\mathrm{E}}\right)^{2}+4\left(\frac{\Delta \overline{\mathrm{v}}}{\overline{\mathrm{v}}}\right)^{2}+9\left[\left(\frac{\Delta \mathrm{a}}{\mathrm{a}}\right)^{2}+\left(\frac{\Delta \mathrm{A}}{\mathrm{A}}\right)^{2}+\left(\frac{\Delta \mathrm{\gamma}}{\gamma}\right)^{2}+\left(\frac{\Delta \rho_{\mathrm{a}}}{\rho_{\mathrm{a}}}\right)^{2}+4\left(\frac{\Delta \mathrm{v}}{\mathrm{v}}\right)^{2}\right.\right. \\
& \left.\left.+x^{2}(\Delta \mathrm{s})^{2}+\mathrm{s}^{2}(\Delta \mathrm{X})^{2}\right]\right\}^{1 / 2}
\end{aligned}
$$


on the assumption that the errors in the various parameters are independent. The known accuracy of the data yields the following rough estimates:

$$
\begin{aligned}
& \frac{\Delta \mathrm{E}}{\mathrm{E}}=0.05 ; \frac{\Delta \overline{\mathrm{V}}}{\overline{\mathrm{V}}}=0.01 ; \frac{\Delta \mathrm{a}}{\mathrm{a}}=0.1 ; \frac{\Delta \mathrm{A}}{\mathrm{A}}=0.15 ; \\
& \frac{\Delta \mathrm{\gamma}}{\gamma}=0.1 ; \frac{\Delta \rho_{\mathrm{a}}}{\rho_{\mathrm{a}}}=0.15 ; \frac{\Delta \mathrm{v}}{\mathrm{v}}=0.003 ; x=0.2 ; \\
& \Delta \mathrm{s}=0.05 ; \mathrm{s}=-0.2 ; \Delta \mathrm{X}=0.1 .
\end{aligned}
$$

By substituting the preceding values into equation (9), we obtain $\Delta=0.77$, in good agreement with the standard deviation of 1 observation equal to \pm 0.80 . This shows that although the individual observational quantities are fairly accurate, our lack of direct knowledge of $A$ and $\gamma$, the variability of the atmospheric density at a given height, and the propagation of the errors cause the large scatter in the values of $\left({ }^{p} / \rho_{m}^{2}\right)_{s}=0$, which is a quantity far remote from the directly observed data.

The dependence of $\left(\log \tau_{\mathrm{p}} / \rho_{\mathrm{m}}^{2}\right)_{\mathrm{s}}=0$ on velocity for our 189 meteors is shown in Table 1. Meteors in short-period orbits are known to be denser than those in long-period orbits (Paper I). Since there is a correlation between velocity and type of orbit, we must account for the consequent variation of $\rho_{\mathrm{m}}$ with velocity before we can determine the value of $n$. A parameter that describes conveniently the type of orbit is the aphelion distance $Q$. Table 2 shows mean values of $\left(\log \tau_{\bar{p}} / \rho_{m}^{2}\right)_{s}=0$ for 7 groups of meteors in order of increasing $Q$. The values of some groups are directly comparable, since their mean velocity is approximately the same; it is immediately apparent that, when $Q<5$ a. u. , 


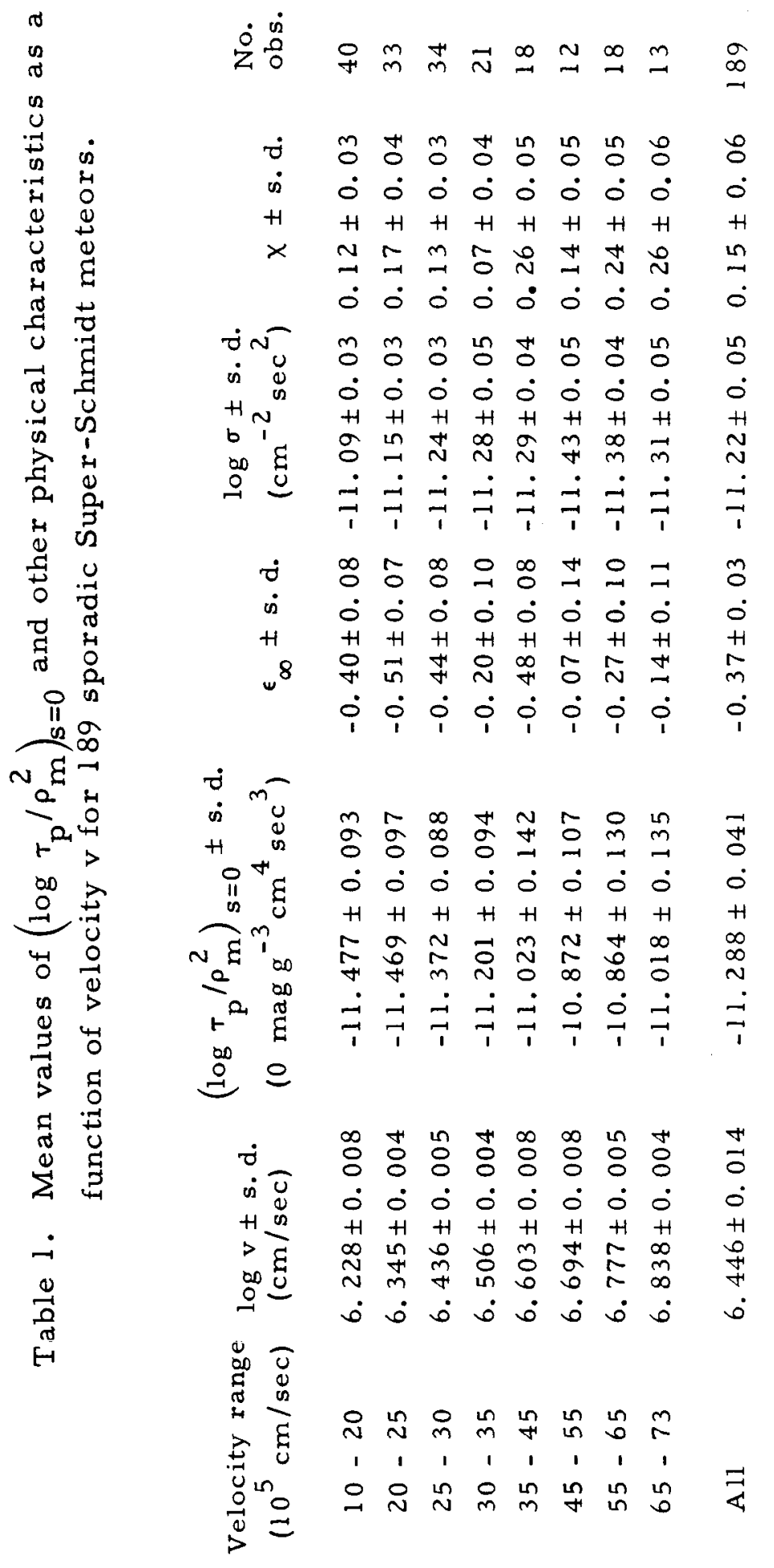

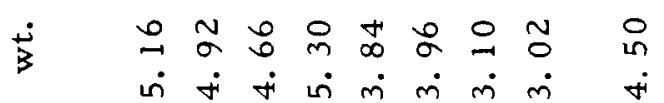

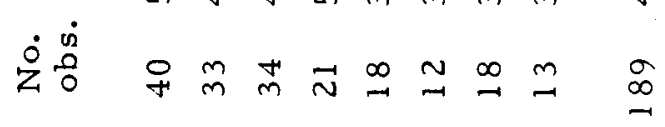

j

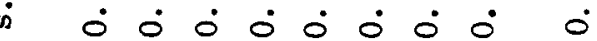

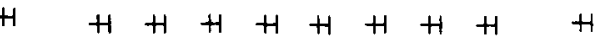

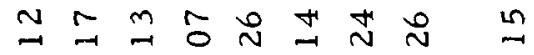

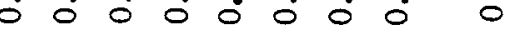

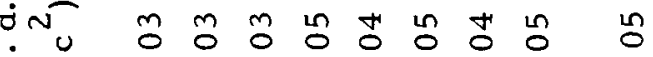

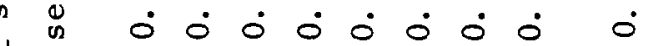

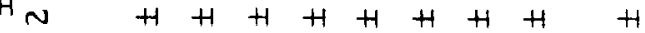

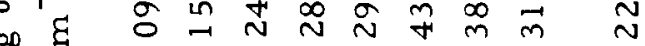

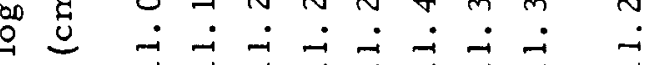

ช $\quad 005$

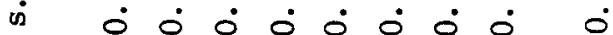

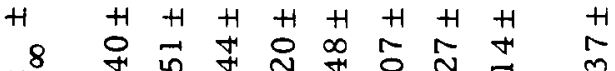

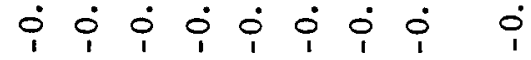



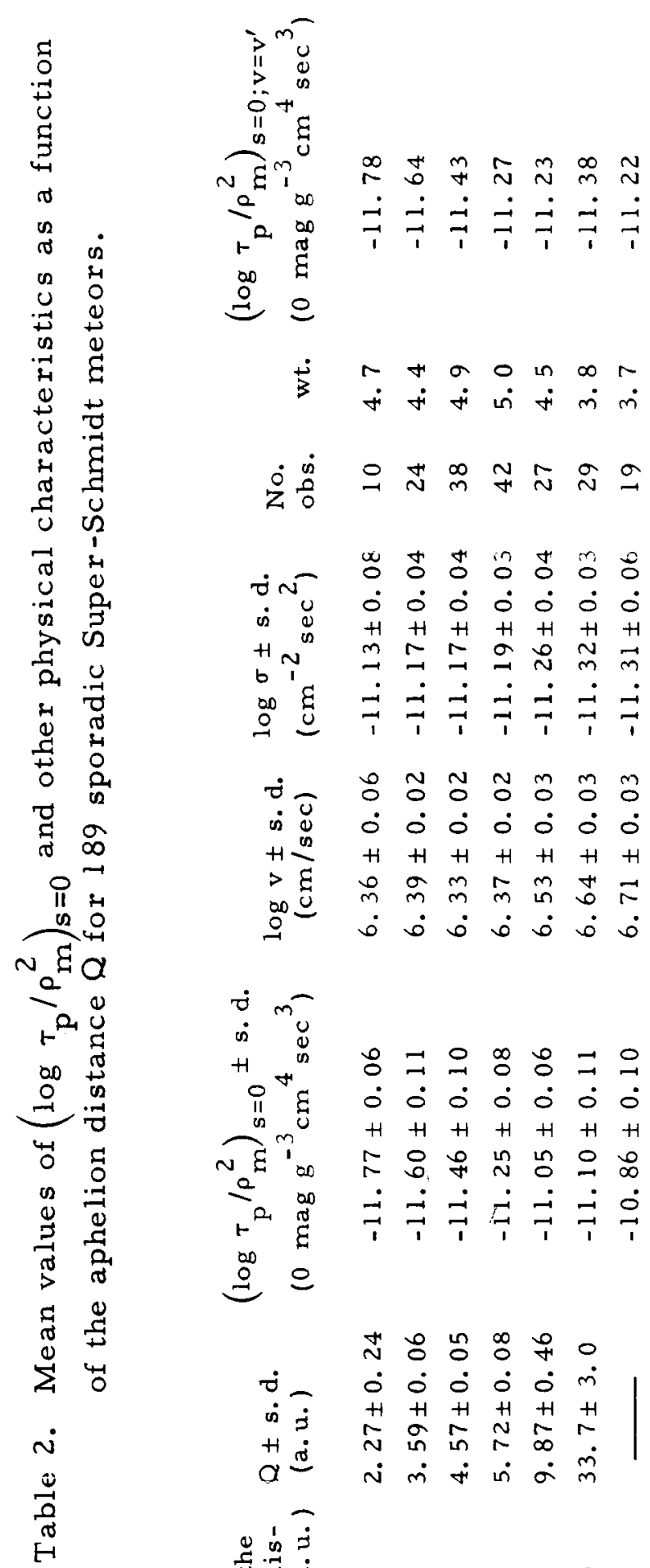

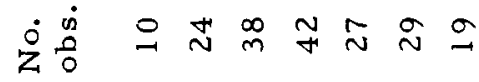

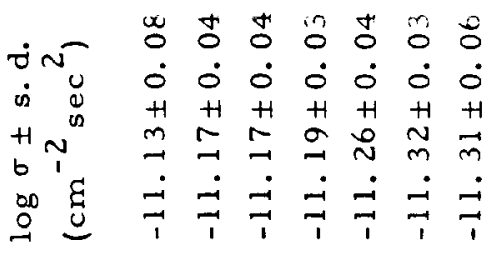

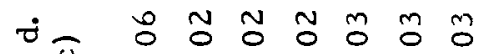

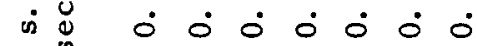

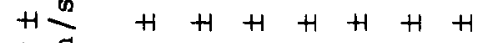

$>$ द्व 0 \% $m$ m $m$ है

$\stackrel{0}{0} \dot{0} \dot{0} \dot{0} \dot{0} \dot{0}$
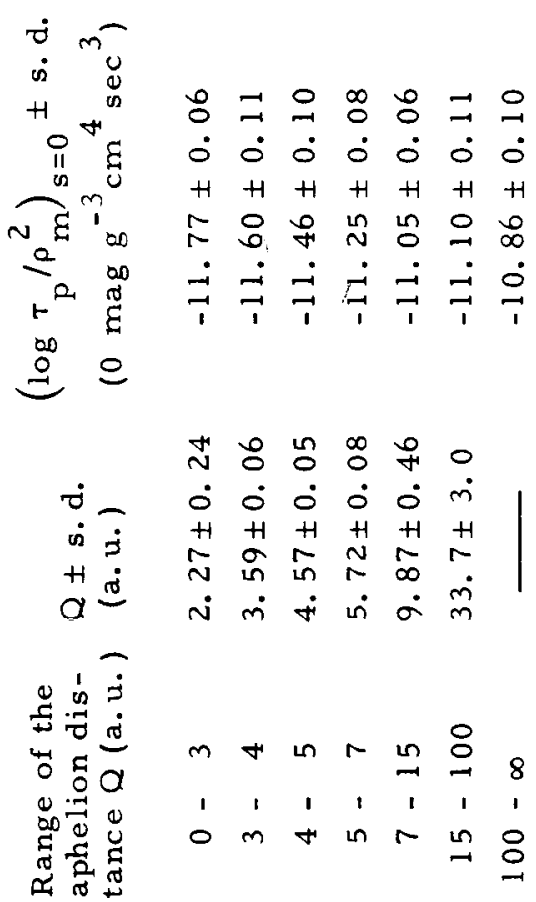
the density increases sharply as $Q$ decreases. We see also that the density of meteors having $Q$ greater than about 10 a. $u$. is approximately constant.

In order to compare all groups, we have to assume a provisional value of $\mathrm{n}$ and then proceed by successive approximations. In the last column of Table 2 , we reduced the values of $\left(\log \tau_{\mathrm{p}} / \rho_{\mathrm{m}}^{2}\right)_{\mathrm{s}}=0$ to $\log$ $\mathrm{v}=6.35$ by assuming provisionally $\mathrm{n}=1$. Dividing the meteors in different groups of increasing $Q$, we find that the transition from the region where $\rho_{m}$ decreases with increasing $Q$ to the region where $\rho_{\mathrm{m}}$ is roughly constant occurs between 5 and $6 \mathrm{a} . \mathrm{u}$. We can very well approximate the dependence of $\left(\log \tau_{p} / \rho_{m}^{2}\right)_{s}=0$ on $Q$ simply with two straight lines:

$$
\begin{array}{ll}
\left(\log T_{p} / \rho_{m}^{2}\right)_{s}=0=-12.252+0.18 Q & Q \leq 5.4 \text { a.u. } \\
\left(\log T_{p} / \rho_{m}^{2}\right)_{s}=0=-11.280, & Q \geq 5.4 \text { a.u. }
\end{array}
$$

By reducing, in turn, the mean values of $\left(\log \tau_{p} / \rho_{m}^{2}\right)_{s}=0$ of the groups of increasing velocity to the density of long-period meteors (i.e., with $Q \geq 5.4$ a.u. ), we obtain the values of Table 3, plotted in Figure 1 . It is apparent from Figure 1 that a straight line fits well all the points, with the exception of the very last one for meteors with $\mathrm{v}>65 \mathrm{~km} / \mathrm{sec}$. A least-squares fit for all points yields $n=0.87$, with a standard deviation of \pm 0.14 . The residual of the last point $(0.18)$ is about 4 times greater than the average and twice as large as the next largest residual. Therefore, it is clear that a better fit for the velocity range 10 to 65 $\mathrm{km} / \mathrm{sec}$ is obtained by disregarding the last point, corresponding to a mean velocity of $69 \mathrm{~km} / \mathrm{sec}$. The result is 
Table 3. Mean values of $\left(\log \tau_{\mathrm{p}} / \rho_{\mathrm{m}}^{2}\right)_{\mathrm{s}=0}$ as a function of velocity, reduced to the density $\rho_{m}^{\prime}$ of the long-period meteors.

\begin{tabular}{|c|c|c|c|c|}
\hline $\begin{array}{l}\text { Velocity range } \\
\left(10^{5} \mathrm{~cm} / \mathrm{sec}\right)\end{array}$ & $\begin{array}{c}\log \mathrm{v} \\
(\mathrm{cm} / \mathrm{sec})\end{array}$ & 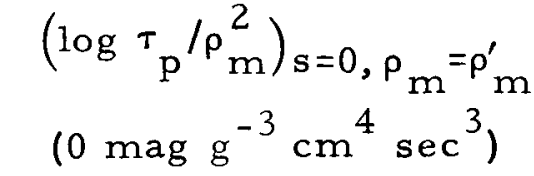 & $\begin{array}{l}\text { No. } \\
\text { Obs. }\end{array}$ & $\begin{array}{c}\text { Total } \\
\text { w. }\end{array}$ \\
\hline $10-20$ & 6.228 & -11.345 & 40 & 206.3 \\
\hline $20-25$ & 6.345 & -11.327 & 33 & 162. 3 \\
\hline $25-30$ & 6.436 & -11.216 & 34 & 158.4 \\
\hline $30-35$ & 6.506 & -11.090 & 21 & 111.3 \\
\hline $35-45$ & 6.603 & -10.963 & 18 & 69.1 \\
\hline $45-55$ & 6.694 & -10.872 & 12 & 47.6 \\
\hline $55-65$ & 6.777 & -10.864 & 18 & 55.8 \\
\hline $65-73$ & 6.838 & -11.018 & 13 & 39.3 \\
\hline
\end{tabular}




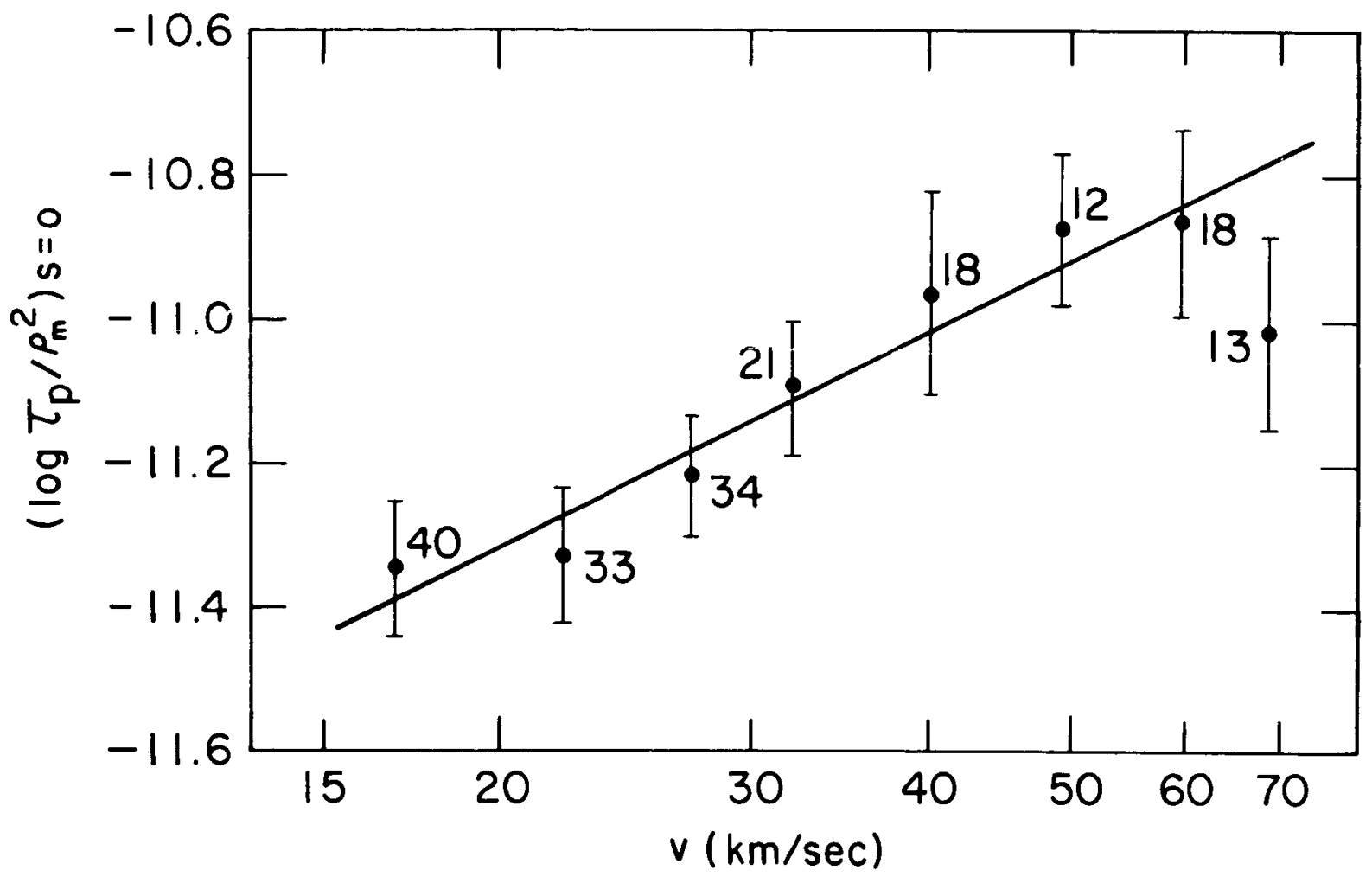

Figure 1. Mean values of $\left(\log \tau_{\mathrm{p}} / \rho_{\mathrm{m}}^{2}\right)_{\mathrm{s}=0}$, reduced to the density of long-period meteors, as functions of velocity for 189 sporadic Super-Schmidt meteors. 


$$
\mathrm{n}=0.99 \pm 0.12
$$

The doubt may arise that the provisional value of $\mathrm{n}$ used to find the dependence of $\rho_{m}$ on $Q$ affects its final determination. To investigate this point, we have repeated the analysis starting with $n=0$. The first approximation yields $n=0.6$; proceeding with successive approximations we finally obtain the same value as in equation (12).

We have so far neglected the possible dependence of $\left(\operatorname{lng} \tau_{\mathrm{p}} / \rho_{\mathrm{m}}^{2}\right)_{\mathrm{s}}=0$ on mass, fragmentation index, and ablation coefficient. The following results justify our procedure. It was shown in Paper I that a dependence of $\tau_{p}$ on mass would produce a dependence on the brightness of the meteor. As a measure of the brightness, we will use

$$
\epsilon_{\infty}=\log E_{\infty}, \quad E_{\infty}=\int_{-\infty}^{+\infty} I_{p} d t .
$$

Table 4 lists average values of $\left(\log \mathrm{T}_{\mathrm{p}} / \rho_{\mathrm{m}}^{2}\right)_{\mathrm{s}}=0$ for groups of meteors in order of increasing $\epsilon_{\infty}$. It is immediately apparent that ${ }^{\top} \mathrm{p} / \rho_{\mathrm{m}}^{2}$ is independent of $\epsilon_{\infty}$ and consequently of mass. The result, together with equation (12), enables us to compute meteor masses as

$$
m_{\infty}=\frac{2}{\tau_{o p}} \int_{-\infty}^{+\infty} I_{p} v^{-3} d t
$$




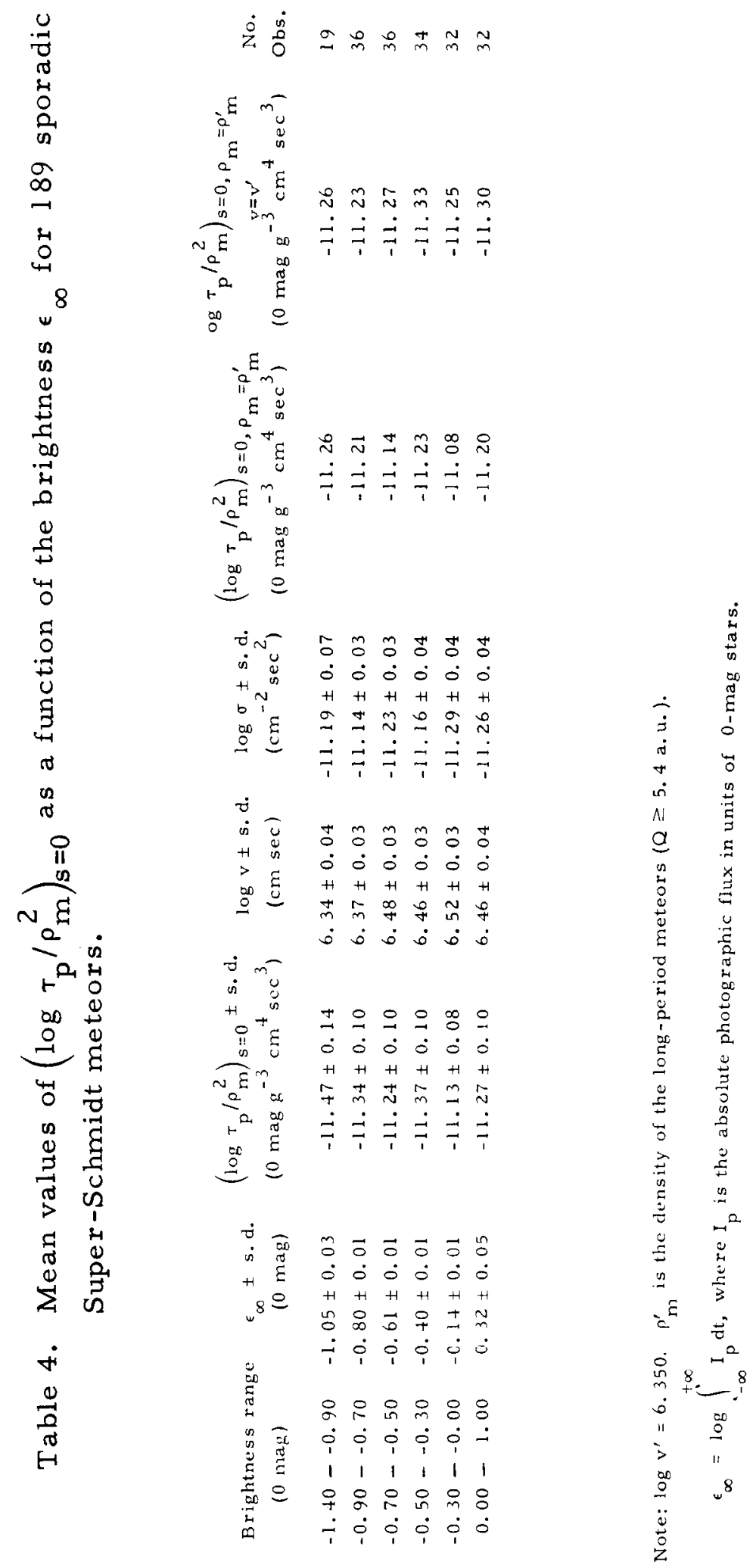


The independence of $\tau_{p}$ on mass is confirmed by Table 5, which shows mean values of $\left(\log \tau_{\mathrm{p}} / \rho_{\mathrm{m}}^{2}\right)_{s}=0$ for groups of meteors with increasing mass. We have assumed provisionally, from Paper $I$, the value $\tau_{\text {op }}=1 \times 10^{-19} 0 \mathrm{mag} \mathrm{g}^{-1} \mathrm{~cm}^{-3} \mathrm{sec}^{4}$.

In Paper I, a correlation was found between $\tau_{p}$ and the fragmentation index $x$ in the sense that nonfragmenting meteors appeared more efficient in producing light than easily crumbling meteors. Most of this correlation was due to the group of meteors with the highest degree of fragmentation (average $x=0.91$ ). The present analysis, limited to meteors with $x<0.7$, does not reveal any correlation between $T_{p}$ and $x$, as can be seen in Table 6. It appears, however, from the results listed in Table 7 that some correlation exists between $\left(\log \tau p / \rho_{m}^{2}\right)_{s}=0$ and the ablation coefficient $\sigma$. Though we could attribute this dependence either to $T_{p}$ or to $\rho_{m}$, it appears much more likely that $T_{p}$ is responsible. In fact, should we assume the opposite, i. e., $\rho_{\mathrm{m}}$ is a function of $\sigma$, the results of Table 7 would show that meteors of higher density have larger values of $\sigma$ and therefore undergo a massloss process more anomalous with respect to the single-body theory than those meteors that are less dense and more crumbly. We are therefore led to admit, as we did in Paper I, that $\tau_{\text {op }}$ increases as $\sigma$ decreases; this means that meteors having light curves in better agreement with the classical theory produce light more efficiently. The determination of the true dependence of $\left(\log \tau_{\mathrm{p}} / \rho_{\mathrm{m}}^{2}\right)_{\mathrm{s}}=0$ on $\sigma$ is made more difficult by the correlation between $\sigma$ and velocity. Once again we have to use a method of successive approximations. The final results, listed in the last columns of Table 7, are plotted in Figure 2 . It is now clear that a part, although small, of the dependence of $\left(\log \tau_{\mathrm{p}} / \rho_{\mathrm{m}}^{2}\right)_{\mathrm{s}}=0$ on velocity is actuaily due to the dependence of $\tau_{\text {op }}$ on $\sigma$. As far as the applications are concerned, this can be safely 


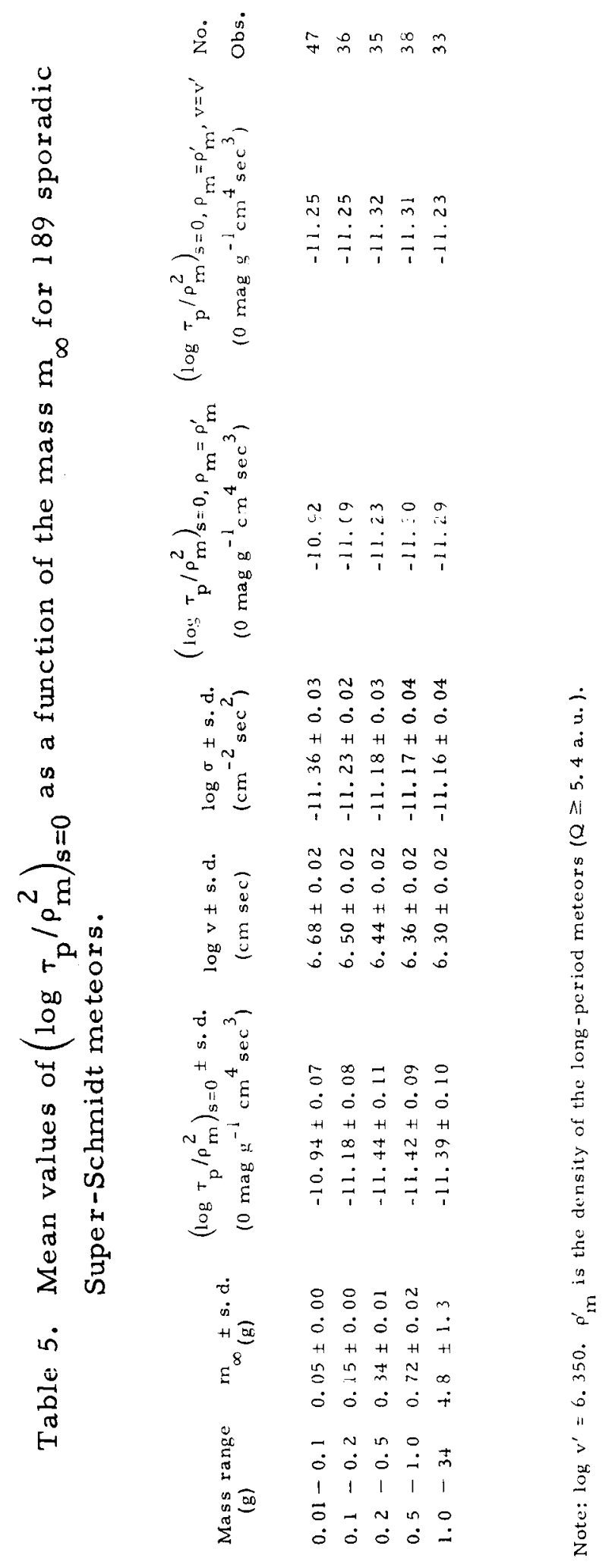




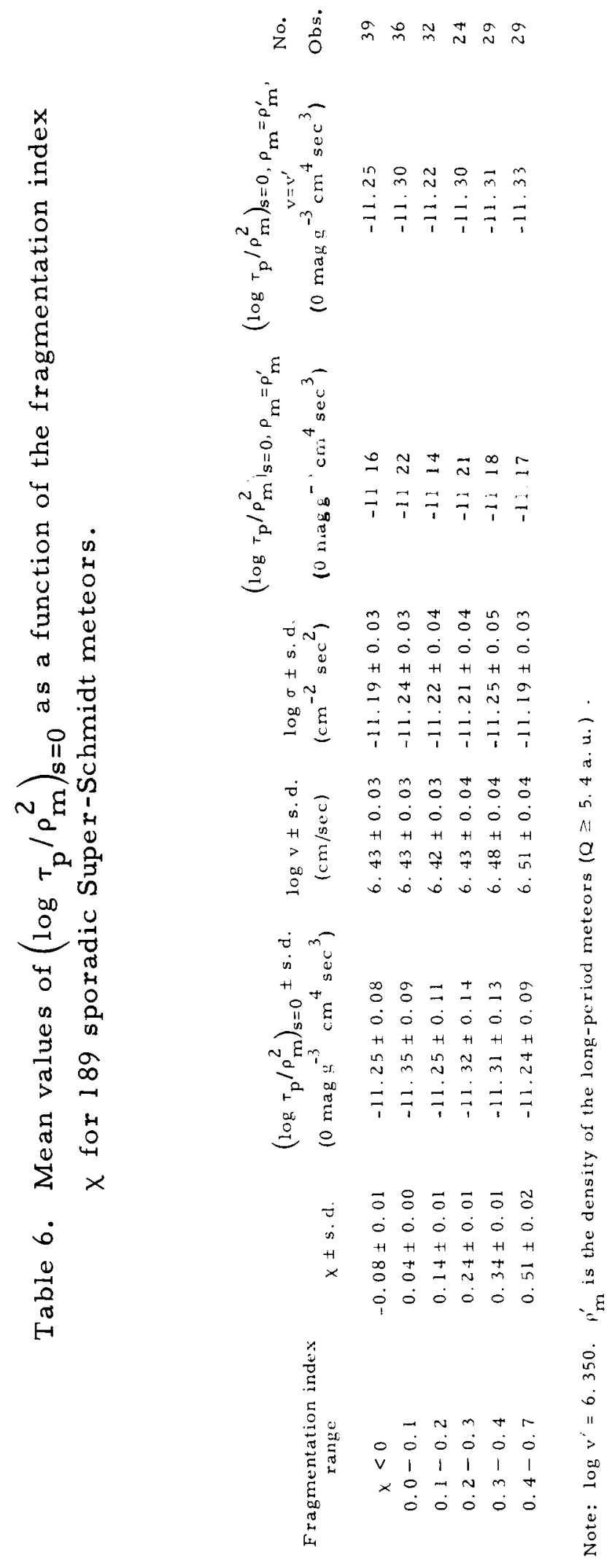




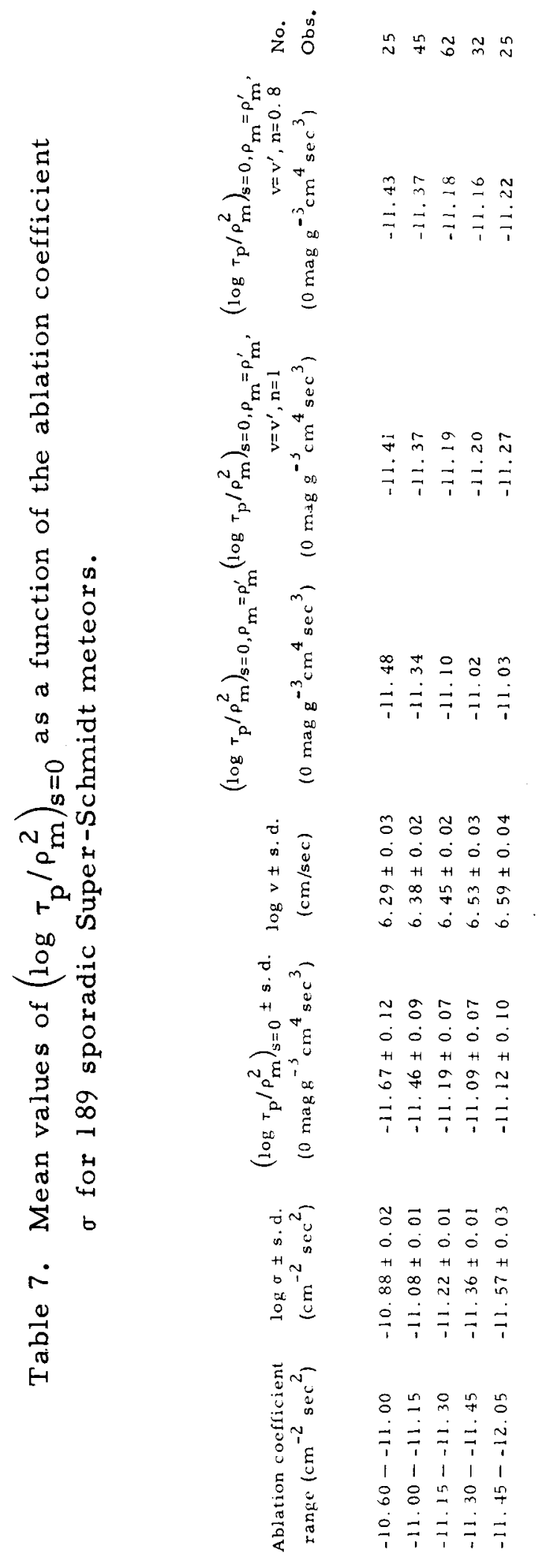



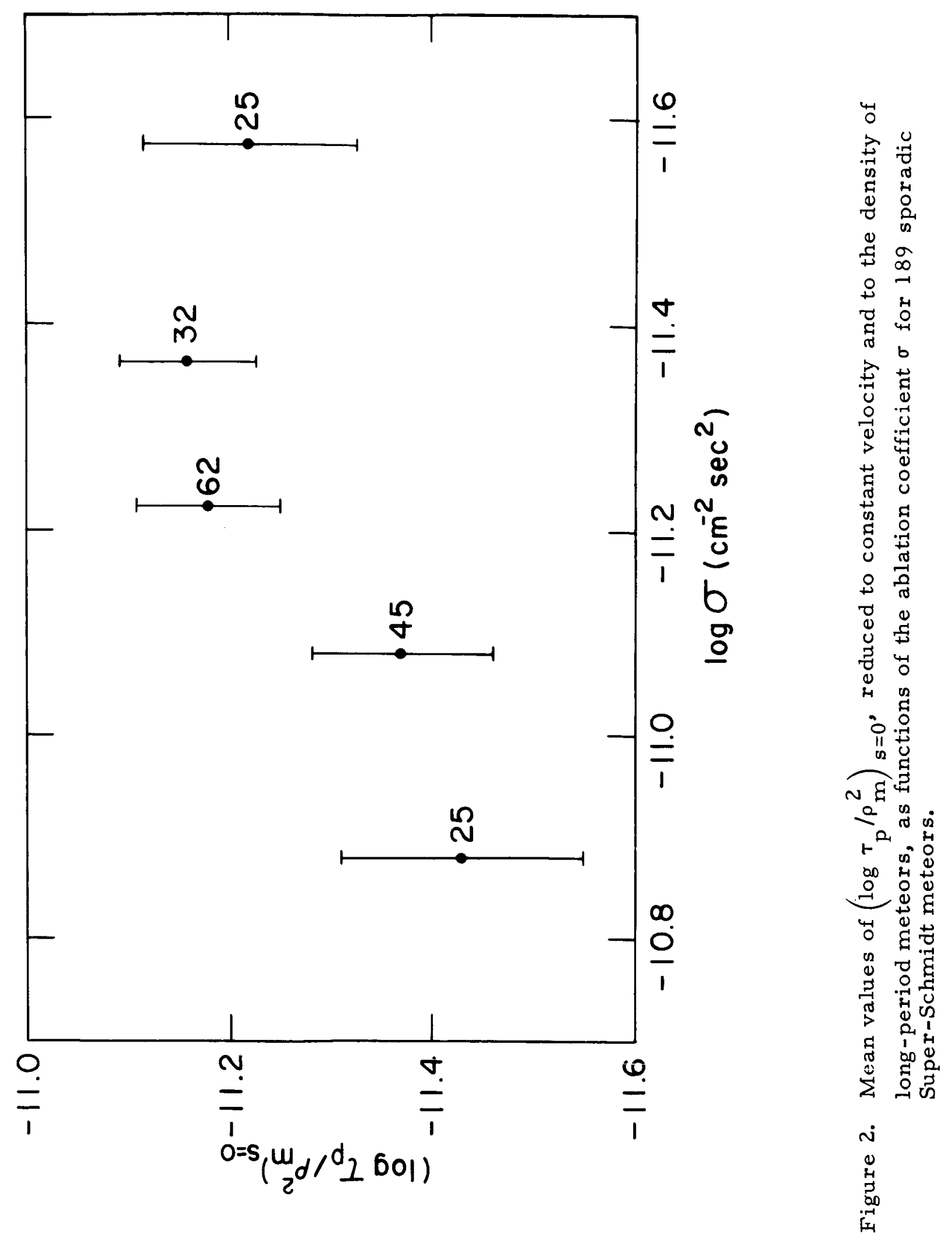
ignored, since it is the complete dependence of $T_{p}$ on $v$ that counts. Should we, however, wish to distinguish between the true dependence on $v$ and that due only to $\sigma$, we find roughly:

$$
\tau_{p} \sim v^{0.76} \sigma^{-0.38}
$$

The probable errors of the two exponents are, respectively, \pm 0.09 for the $\mathrm{v}$ exponent and \pm 0.12 for the $\sigma$ exponent.

Since $\sigma$ varies only slightly with $Q$, we would not expect that the use of equation (14) to reduce the values of $\left(\log \tau_{p} / \rho_{m}^{2}\right)_{s}=0$ to the constant density of meteors with long-period orbits would make any difference in the final value of $n$. A check has nevertheless been made and the result has shown that no change in the final value of $n$ occurs. The value $\mathrm{n}=1.0$ can therefore be considered well established. The standard deviation \pm 0.12 corresponds to a probable error of \pm 0.08 , one half of the probable error of the determination made in Paper $I$. In view of the irregular dependence of $\tau_{p}$ on $\sigma$ (see Fig. 2), the constant exponent of $\sigma$ in equation (14) provides only a rough approximation; for practical applications it is advisable to use only the apparent dependence of $\tau_{p}$ on $v$ as expressed by $\tau_{p}=\tau_{o p} v$. 


\section{THE LUMINOUS EFFICIENCY COEFFICIENT $\tau_{\text {op }}$}

In Paper I the author suggested the adoption of the value $\tau_{\text {op }}=1 \times 10^{-19} 0 \mathrm{mag} \mathrm{g} \mathrm{cm}^{-1} \mathrm{sec}^{4}$ for computing meteor masses by means of equation (13). This value, extensively used since then, was based only on the data of one asteroidal meteor (Harvard Meteor No. 1242 ) and on the extrapolation of the results from the artificial meteors of McCrosky and Sobcrman (1963). To these we can add now the results from two other asteroidal meteors, namely, Harvard Meteors No. 7946 and No. 19816.

Meteor No. 7946 provides the most reliable data. The complete set of observational data for this meteor has been recently published by Jacchia, Verniani, and Briggs (1965). These authors have also discussed the evidence for its asteroidal origin. Here we shall recall only that the deceleration of this meteor was determined at 10 different points of its trajectory and that a single fragment became detached from the main body a little before midtrajectory and was followed on the photograph for $0.1 \mathrm{sec}$. Up to the point when the fragment became detached (at $s=-0.07$ ), the meteor did not show any sign of progressive fragmentation and $x$ is zero. After the detachment of the fragment, however, progressive fragmentation appeared to take place and $\chi$ increased appreciably as is shown in Figure 3, where the logarithm of the ratio $\mathrm{a} / \mathrm{a}_{\mathrm{T}}$ is plotted against the mass-loss parameter $\mathrm{s}$. Two straight lines provide a very good fitting of the observational points. The first, horizontal $(x=0)$, fits from the beginning to $s \simeq-0.05$; the second, from $s \simeq-0.05$ to the end, with a slope $X=0.31$. Since $X$ is zero in the first half of the trajectory, the reduction of the values of $\tau_{\mathrm{p}} / \mathrm{p}_{\mathrm{m}}^{2}$ to a given value $s_{0}$ of $s$ (of course, $s_{0} \leq-0.05$ ) has a full physical meaning . 

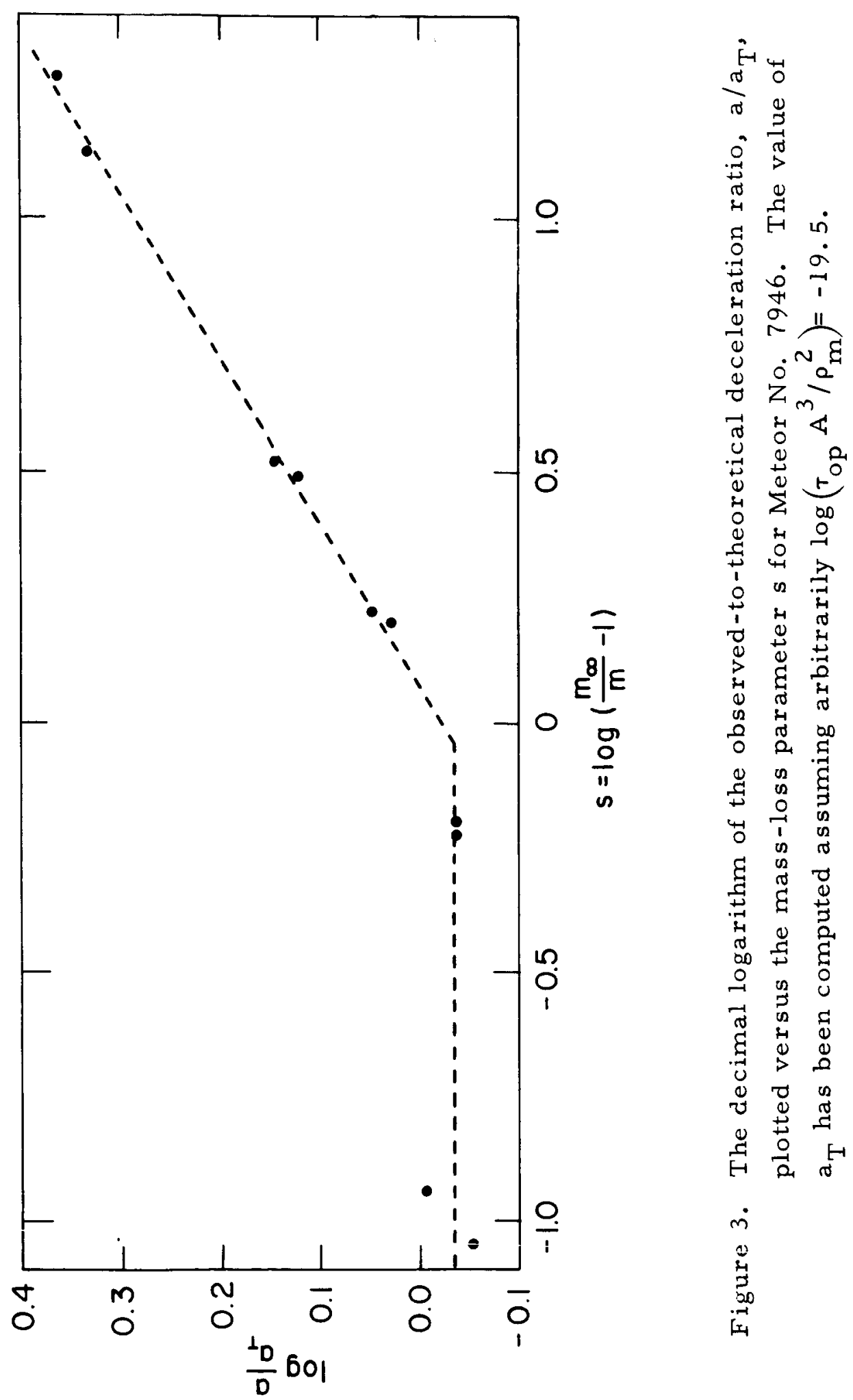
This asteroidal meteor is compact and fairly large: the use of $\gamma=1.1$ would not be justified at all. We have computed its $\gamma$ from A. F. Cook's (1966) formula

$$
\gamma=0.55\left[1+8.3 \times 10^{-5}\left(\mathrm{r} \rho_{\mathrm{a}}\right)^{-1 / 2}\right],
$$

where $r$, the equivalent radius of the meteoroid, and $\rho_{a}$ a re expressed in cgs units. The radius $r$ was computed from the observed brightness, assuming $\tau_{o p}=1 \times 10^{-19} 0 \mathrm{mag} \mathrm{g}^{-1} \mathrm{~cm}^{-3} \mathrm{sec}^{4}$ and $\rho_{\mathrm{m}}=3.5$ $\mathrm{g} \mathrm{cm}^{-3}$. Since $\mathbf{r}$ is proportional to $\left(\tau_{\mathrm{op}} \rho_{\mathrm{m}}\right)^{-1 / 3}$, the term $\mathrm{r}^{-1 / 2} \sim$ $\left(\tau_{o p} \rho_{m}\right)^{1 / 6}$ is quite insensitive to the uncertainty in $\tau_{\text {op }} \rho_{m}$. It can be shown that an error in $\tau_{o p} \rho_{m}$ of a factor of 10 produces an error in $\gamma$ of only 10 percent. The values of $\log \left(\tau_{\mathrm{op}} \mathrm{A}^{3} / \mathrm{\rho}_{\mathrm{m}}^{2}\right)$, reduced to $\mathrm{s}=\mathrm{s}_{0}$ $\left(s_{0} \leq-0.05\right)$, range from -19.67 to -19.52 . The arithmetical average is -19.606 , with a standard deviation of \pm 0.012 . Weights have not been used, since the accelerations are all very good and the corrections for fragmentation can be computed accurately also in the last part of the trajectory. The terminal mass $\mathrm{m}_{\mathrm{E}}$ of this meteor is completely negligible, as can be seen by use of the integrated form of the mass equation:

$$
\frac{m_{E}}{m_{\infty}}=\exp \left[-\frac{\sigma}{2}\left(v_{\infty}^{2}-v_{E}^{2}\right)\right]
$$

The data concerning Meteor No. 19816 have been published by Cook, Jacchia, and McCrosky (1963). The value of the total integrated brightness, not given in the publication, has been furnished by McCrosky (1966) in the form $\tau_{o p}\left(m_{\infty}-m_{E}\right)=1.446 \times 10^{-18} 0 \mathrm{mag} \mathrm{cm}^{-3} \mathrm{sec}^{4}$. Eight deceleration 
solutions were computed for this meteor. "During the final portion of the trajectory, two particles are in evidence": the splitting occurs when $s$ is approximately -0.1 . "The deceleration history of the separate particles indicates that they have nearly comparable masses," and therefore account must be taken of the increase of the cross-sectional area by a factor of $2^{1 / 3}$ when two particles are present. "It appears that the terminal mass is neglibible, as would be expected for a meteor with an initial velocity of $21 \mathrm{~km} \mathrm{sec}{ }^{-1}$." (The quotations are from Cook, Jacchia, and McCrosky, 1963.) The values of $\log a / a_{T}$ versus s, plotted in Figure 4, show an irregular pattern. Contrary to what was expected, Figure 4 shows signs of more progressive fragmentation at the beginning than at the end. By forcing a straight line fit to the points of Figure 4, we obtain $x=0.09 \pm 0.04$. Without any reduction to a given value of $s$, the values of $\log \left(\tau_{\mathrm{op}} \mathrm{A}^{3} / \mathrm{\rho}_{\mathrm{m}}^{2}\right)$ range from -19.88 to -19.00 , with an average of -19.37 and a standard deviation (of the mean) of \pm 0.11 . If we reduce the values of $\log \left(\tau_{\mathrm{op}} \mathrm{A}^{3} / \mathrm{p}_{\mathrm{m}}^{2}\right)$ to $s=0$, the new values range from -19.77 to -19.11 ; the average is $-19.39 \pm 0.09$. It is evident, however, that in this case the reduction to $s=0$ has little meaning, since even in the early portion of the trajectory $x$ appears to be positive. [Should we reduce all the values of $\log \left(\tau_{o p} A^{3} / \rho_{m}^{2}\right)$ to $s=-1, i . e$, to a point close to the actual beginning of the meteor, we would obtain $\log \left(\tau_{\mathrm{op}} \mathrm{A}^{3} / \mathrm{\rho}_{\mathrm{m}}^{2}\right)=$ -19.65.] The result obtained from this meteor is far more uncertain than the one obtained from Meteor No. 7946.

The observational data for Meteor No. 1242 were originally published by Jacchia (1949), who computed the meteor deceleration at four points of the trajectory. Using these data and neglecting the terminal mass, we found in Paper I $\log \left(\tau_{\text {op }} A^{3} / p_{m}^{2}\right)=-19.91$. Cook, Jacchia, and MícCrosky(1363) did not use Jacchia's four original solutions, but recomputed the deceleration at six different points. By assuming the quantity $\left(\tau_{o p} \gamma^{3} A^{3} / \rho_{m}^{2}\right)$ to be constant along the trajectory, grouping the first 

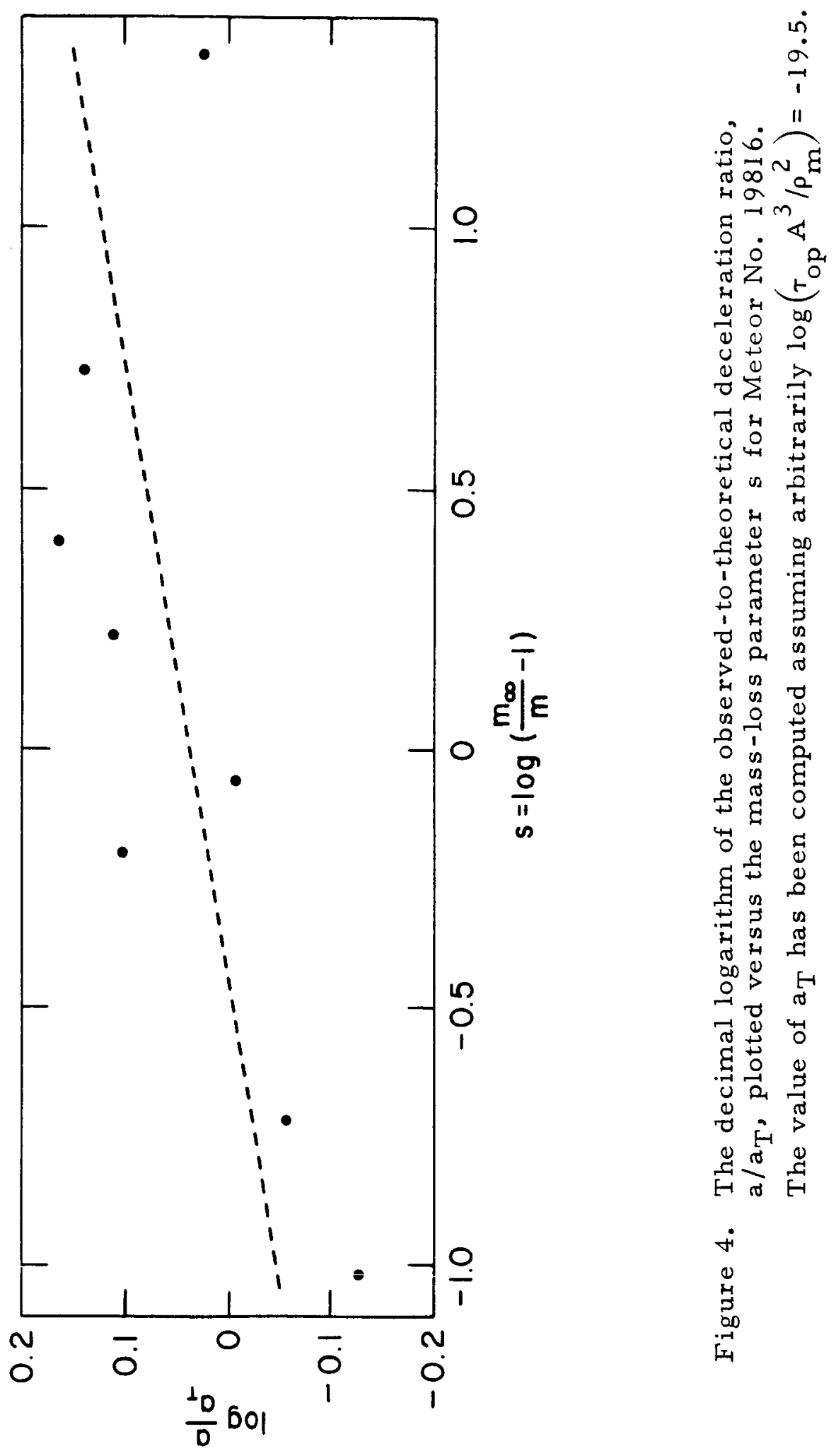
three epochs and the last three epochs, and using average values for each group, they found that the terminal mass of the meteor should have been about one-half its initial value. However, if we compute the terminal mass by means of equation (16) by assuming $v_{E}=6 \mathrm{~km} \mathrm{sec}^{-1}$ and by using successive approximations, we find a value of the order of one-tenth the initial mass. Should we assume for $v_{E}$ a smaller value, e.g., $v_{E}=4 \mathrm{~km} \mathrm{sec}^{-1}$ as suggested by McCrosky and Soberman (1963), $\mathrm{m}_{\mathrm{E}}$ might be even smaller. Cook, Jacchia, and McCrosky (1963) used the ARDC 1959 atmosphere (Minzner, Champion, and Pond, 1959); the same calculation with the U.S. Standard Atmosphere (1962) yields $\mathrm{m}_{\mathrm{E}} / \mathrm{m}_{\infty}=0.44$. However, since Figure 5 shows that the first acceleration is somewhat anomalous, it seems reasonable to repeat the computation of Cook, Jacchia, and McCrosky (1963) with only the other five accelerations. The result is $\mathrm{m}_{\mathrm{E}} / \mathrm{m}_{\infty}=0.74$. Should we discard also the last deceleration, we would obtain a negative value for the terminal mass. In conclusion, the method used by Cook, Jacchia, and McCrosky (1963) appears to yield results too much dependent on individual accelerations, and therefore seems less reliable than the direct approach of equation (16). We shall adopt $m_{E} / m_{\infty}=0.1$. The values of $\log \left(\tau_{o p} A^{3} / \rho_{m}^{2}\right)$ computed from Jacchia's original data range from -19.61 to $-20.05 \mathrm{cgs}$ units, with an average of -19.86; those computed from the data of Cook, Jacchia, and McCrosky (1963) range from -19.62 to -20.03 , with an average of -19.81 . The mean of all 10 values is $-19.83 \pm 0.05$. The values of $\log a / a_{T}$ plotted in Figure 5 show that a reduction to a given value of $s$ is not necessary. If we had adopted $m_{E} / m_{\infty}=0.3$, the mean value of $\log \left(\tau_{o p} A^{3} / \rho_{m}^{2}\right)$ would have been -19.62 .

Cook, Jacchia, and McCrosky (1963) also took into consideration Halliday's (1960)iron meteor (known as spectrum no. 2i 0) and obtained a value of the luminous efficiency coefficient foriron. However, since the deceleration and thelight curve of that meteor were not available, they had to compute the total emitted light from the panchromatic magnitude, by using a simple approximation of the classical theory, quite similar to 


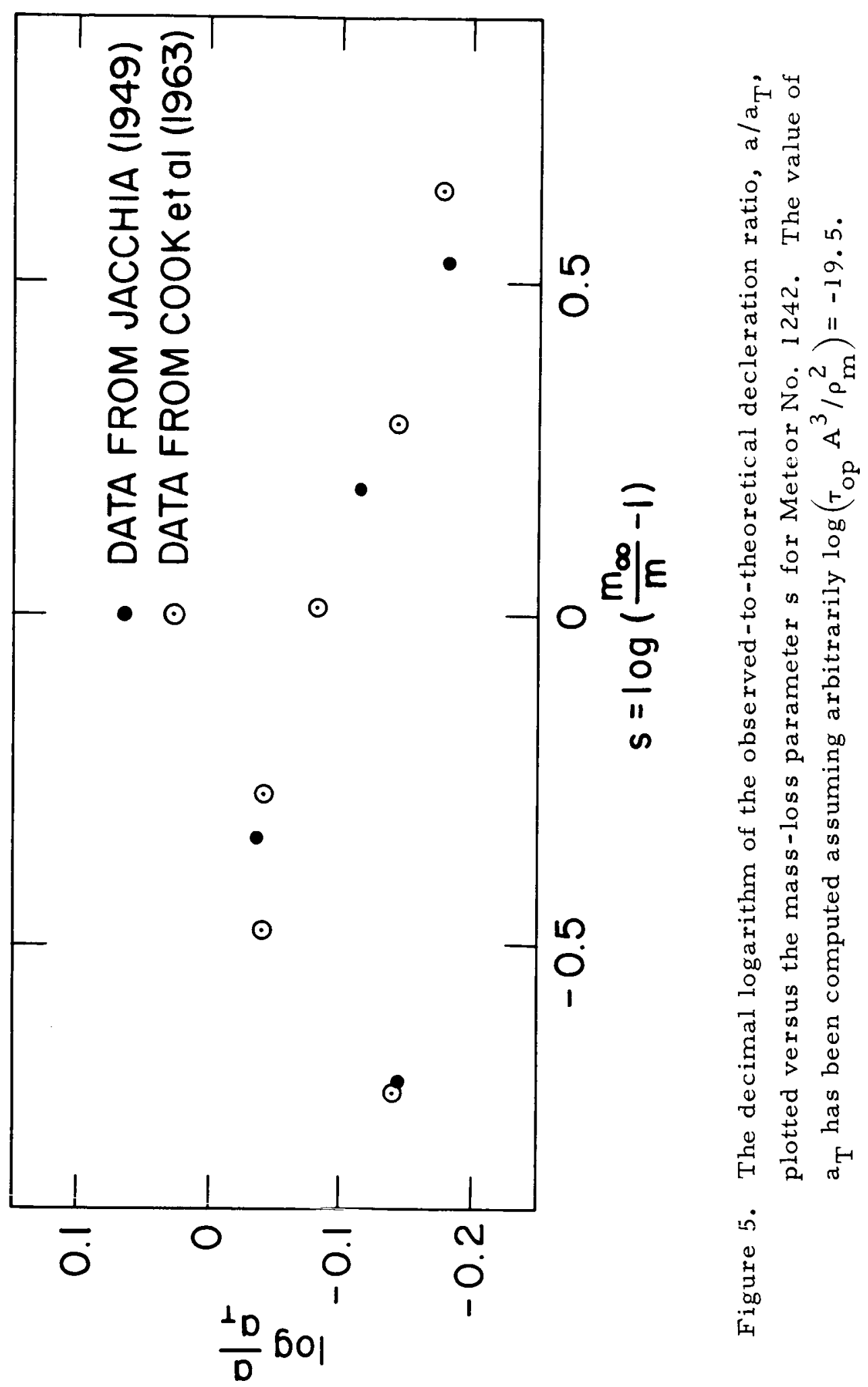


Herlofson's (1948) treatment. They also computed upper and lower limits for the radius of the particle, making use again of the approximate theory and with the help of several assumptions. Consequently, the uncertainty in the value of the luminous efficiency that can be obtained from spectrum no. 210 is much larger than that for the meteors previously discussed. We will not use spectrum no. 210 at all for our determination of $T_{\text {op }}$. Ceplecha, Rajchl, and Sehnal (1959) published complete data for a very bright mete or (absolute visual magnitude $\sim-6$ ) whose orbit, in the author's words, is typically asteroidal. The completeness of the published data allows us to compute as many as 20 values of $\log \left(\tau_{\text {op }} A^{3} / \rho_{m}^{2}\right)$. The meteor shows a value of $X$ very close to zero. Unfortunately, the value of $\log \left(\tau_{\text {op }} A^{3} / \rho_{m}^{2}\right)$ is -17.87 , typical of cometary meteors.

Summing up the results obtained in this section, we see that the best value for $\log \left(\tau_{\text {op }} \mathrm{A}^{3} / \mathrm{\rho}_{\mathrm{m}}^{2}\right)$ is -19.6 . In view of the exceptional reliability of the result from meteor no. 7946 , we estimate the uncertainty as being of the order of \pm 0.1 . Stony meteorites greatly outnumber the irons. It is therefore statistically very likely that our three asteroidal meteors were made of stone. The density of stony meteorites ranges generally from 3.3 to $3.9 \mathrm{~g} \mathrm{~cm}^{-3}$, with an average of about $3.6 \mathrm{~g} \mathrm{~cm}^{-3}$ (Wood, 1963). The most reasonable average value for the shape factor $A$ is 1.5 , with an uncertainty of the order of 10 percent. Consequently, we obtain $\log \tau_{\text {op }}=-19.02$ with an uncertainty of \pm 0.15 . This result confirms for the asteroidal meteors the value proposed by the author in Paper I:

$$
\tau_{\text {op }}=1.0 \times 10^{-19} 0 \mathrm{mag} \mathrm{g} \mathrm{g}^{-1} \mathrm{~cm}^{-3} \mathrm{sec}^{4} \text {, }
$$


but the uncertainty is reduced to 30 or 40 percent of the value itself. This value is also in good agreement with the value extrapolated by McCrosky and Soberman (1963) from their artificial meteors.

The question naturally arises, how does the value of $\tau_{\text {op }}$ obtained for asteroidal meteoroids apply to cometary particles, the most common bodies by far, but also the least known? Whipple (1963) does not explicitly raise the question but implicitly assumes for cometary meteors the same value found for asteroidal stone. In view of the lack of direct information, this seems to be the only reasonable procedure, since the abundance of nonvolatile elements in the earth and in the sun, which is representative of cometary materials, is roughly the same as the abundance found in stony meteorites, which are representative of asteroidal stones.

In conclusion, we have for asteroidal stones, and probably also for cometary meteors:

$$
\tau_{p}=1.0 \times 10^{-19} \mathrm{v}
$$

in cgs and 0-mag units. In normal cgs units, equation (18) is equivalent to (see Paper I):

$$
T_{p}=5.25 \times 10^{-10} \mathrm{v},
$$

where $\tau_{p}$ expresses the ratio between the energy (in ergs) radiated in the blue-emulsion spectral range by the atoms ablated from the meteoroid in a time interval $\Delta t$ and the kinetic energy lost by the same atoms. 
Equation (18) confirms the result obtained by Jacchia, Verniani, and Briggs (1965) that the initial mass of a zero visual magnitude meteoroid (color index $=-1.86$ ) with a velocity of $40 \mathrm{~km} \mathrm{sec}^{-1}$ and a zenith angle of $50^{\circ}$ is $0.76 \mathrm{~g}$. The visual luminous efficiency can be obtained from equation (18) and the color index curve given in Jacchia, Verniani, and Briggs's (1965) Figure 12.2. The ionizing efficiency $\tau_{q}$, found by Verniani and Hawkins (1964) on the basis of the photographic luminous efficiency of Paper I, is also confirmed. In cgs units it is

$$
\tau_{\mathrm{q}}=6 \times 10^{-17} \mathrm{v}^{2},
$$

with an uncertainty of about 50 percent in the coefficient and 20 percent in the velocity exponent. 


\section{METEOR DENSITIES}

Once the mass scale has been established by determining the luminous efficiency, we can easily evaluate the meteor densities. The density distribution for the 220 sporadic meteors discussed in Section 2 is shown in Figure 6. The weighted mean of $\log \rho_{m}$ is $-0.558 \pm 0.025$, corresponding to a logarithmic mean density of $0.28 \mathrm{~g} \mathrm{~cm}^{-3}$, with a standard deviation of 6 percent. The mean weighted density is $(0.42 \pm 0.04) \mathrm{g} \mathrm{cm}^{-3}$, but it is not a representative average because of the effect of the large individual values of $\rho_{\mathrm{m}}$. The weighted median is $0.23 \mathrm{~g} \mathrm{~cm}^{-3}$. The arithmetic averages are very close to the weighted ones.

We have seen in Section 2 that because of the several uncertainties in the computations and the error propagation, the standard deviation expected in the individual value of $\left(\log \tau_{\mathrm{p}} / \rho_{\mathrm{m}}^{2}\right)_{\mathrm{s}=0}$ is about \pm 0.8 , in good agreement with the observed standard deviation of one observation. This agreement means that the presence of a large scatter eithe $r$ in the true values of $\tau_{p}$ or in those of $P_{m}$ is unlikely. We already know, however, from Section 2 that the density $P_{m}$ is a function of the aphelion distance $Q$, the average density of a meteor with $Q=2 \mathrm{a}$. $\mathrm{u}$. being about 50 percent larger than the average density of a meteor with $Q=4 \mathrm{a}$. $\mathrm{u}$. The mean logarithmic density as a function of the aphelion distance is shown in Table 8 and plotted in Figure 7. Figure 7 shows clearly that the logarithmic spread in $\log P_{m}$ due to the orbital dependence is of the order of only 0.1 . Consequently, it is not large enough to affect the observed spread due to the error propagation; the total scatter is the square root of the sum of the squares of each error. For this reason, since the errors due to atmospheric variability, changes of shape, and 


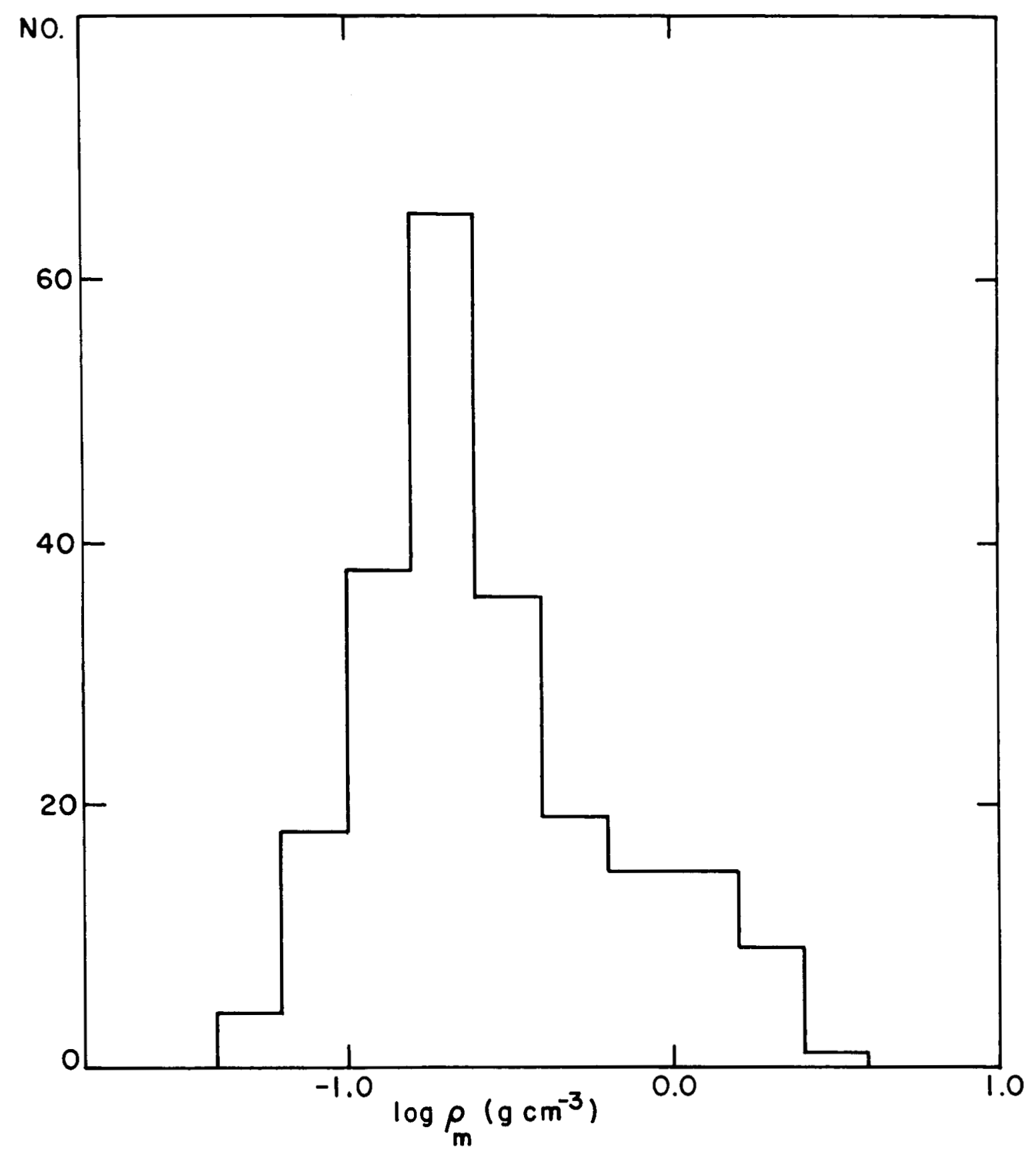

Figure 6. The density distribution for 220 sporadic Super-Schmidt meteors. 
Table 8. The mean logarithmic density as a function of the aphelion distance, Q, for 220 sporadic Super-Schmidt meteors.

\begin{tabular}{|c|c|c|c|c|}
\hline $\begin{array}{l}\text { Range of } Q \\
\quad(a . \text { u. })\end{array}$ & $\begin{array}{l}\text { Mean Q } \\
(\text { a.u. })\end{array}$ & Mean $\underset{(\mathrm{g} / \mathrm{lom})}{\rho_{\mathrm{m}}} \pm$ s.d. & S.d. of 1 obs. & No. obs. \\
\hline $0.9-3$ & 2.20 & $-0.184 \pm 0.099$ & \pm 0.40 & 16 \\
\hline $3-4$ & 3.63 & $-0.381 \pm 0.068$ & \pm 0.37 & 30 \\
\hline $4-5$ & 4.56 & $-0.515 \pm 0.060$ & \pm 0.40 & 45 \\
\hline $5-6$ & 5.41 & $-0.690 \pm 0.055$ & \pm 0.30 & 31 \\
\hline $6-10$ & 7.24 & $-0.649 \pm 0.045$ & \pm 0.24 & 29 \\
\hline $10-30$ & 16.27 & $-0.632 \pm 0.061$ & \pm 0.32 & 27 \\
\hline $30-100$ & 44.84 & $-0.672 \pm 0.082$ & \pm 0.38 & 21 \\
\hline $100-\infty$ & $766 *$ & $-0.690 \pm 0.069$ & \pm 0.31 & 21 \\
\hline All & - & $-0.558 \pm 0.025$ & \pm 0.37 & 220 \\
\hline
\end{tabular}



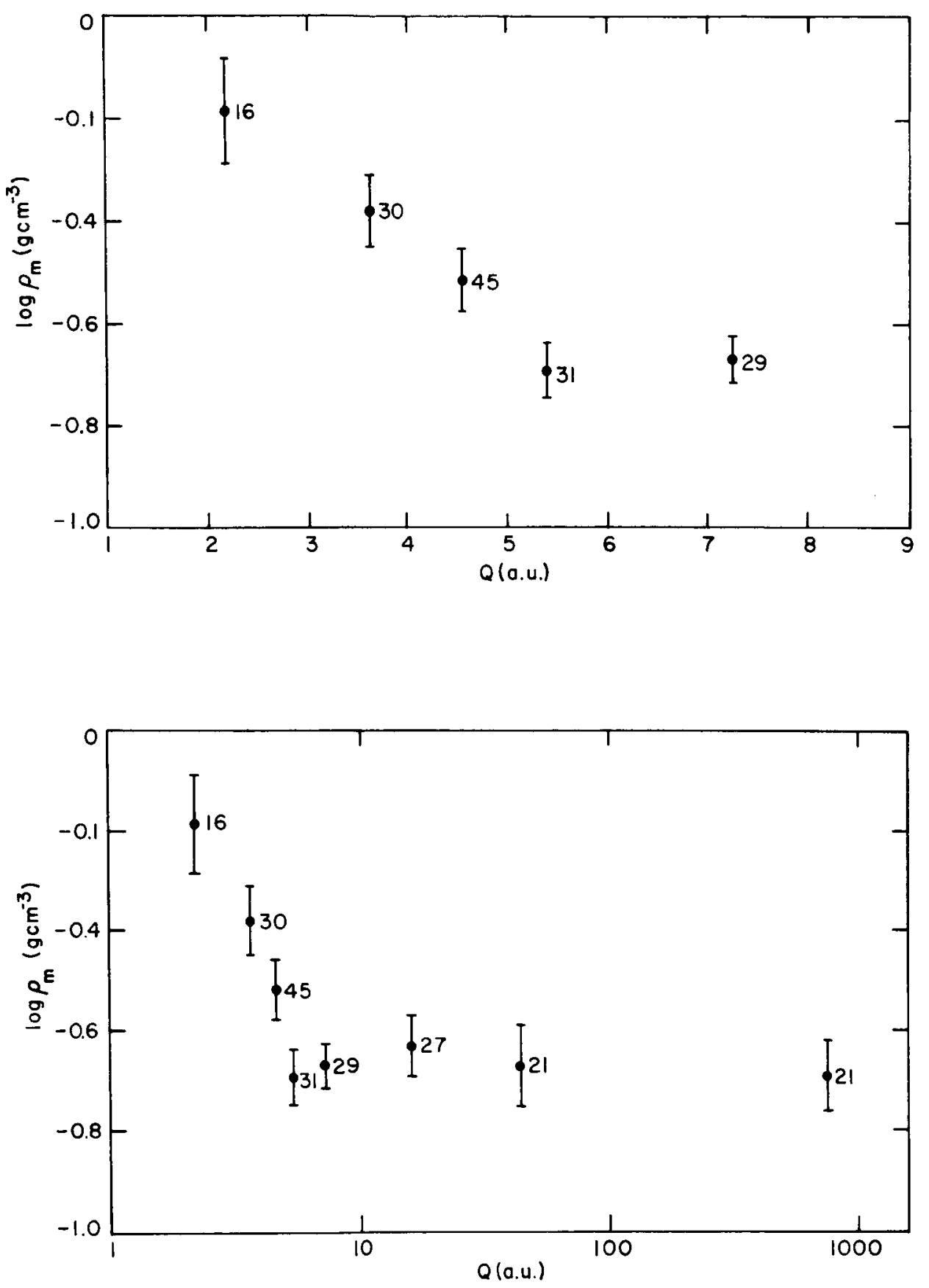

Figure 7. The density of 220 sporadic Super-Schmidt meteors, as function of the aphelion distance $Q$. 
observational inaccuracies are random, we would expect a Gaussian distribution for $\log P_{m}$ centered around the logarithmic mean. Figure 6 shows, on the contrary, that the $\log \rho_{\mathrm{m}}$ distribution extends itself much more on the high-densities side. In Figure 8 the same meteors have been divided according to their aphelion distance, and the density distribution is shown separately for meteors with $Q<5.4$ a. u. and $Q>5.4$ a. u. It is immediately apparent that the density distribution of the long-period meteors is Gaussian. A X-square test confirms that the distribution obtained is fully compatible with a Gaussian curve centered around the logarithmic mean and with the dispersion fixed by the observed standard deviation. The medil (weighted) logarithmic density for these long-period sporadic meteors is $0.21 \mathrm{~g}$ $\mathrm{cm}^{-3}$, with a standard deviation of 7 percent. The median is also $0.21 \mathrm{~g} \mathrm{~cm}^{-3}$.

The distribution of the short-period meteors is different. The low- and middle-density regions are quire similar to the corresponding ones in the distribution of long-period meteors. Conversely, on the high-densities side it extends considerably, and it looks as though there is an overlapping of two Gaussian distributions, one centered around $\log \rho_{\mathrm{m}}=-0.7$, as for the long-period meteors, and the other centered around $\log \rho_{\mathrm{m}}=0.1$. We are thus led to the conclusion that a small group of short-period meteors may have densities of the order of $1 \mathrm{~g}$ $\mathrm{cm}^{-3}$. Figure 9 shows the density distributions for three subgroups of short-period meteors. It is immediately apparent that the group with the smallest aphelion distances $(Q<3$ a. u. ) includes the largest percentage of higher density meteors: 50 percent of the total with log $P_{m}>-0.2$. The other two subgroups contain a much smaller percentage of high-density meteors (around 20 or 25 percent); it is apparent also that the average density of the majority group is shifted systematically toward higher values when we pass from a subgroup with a greater $Q$ to one with a smaller $Q$. The latter fact shows that the dependence 

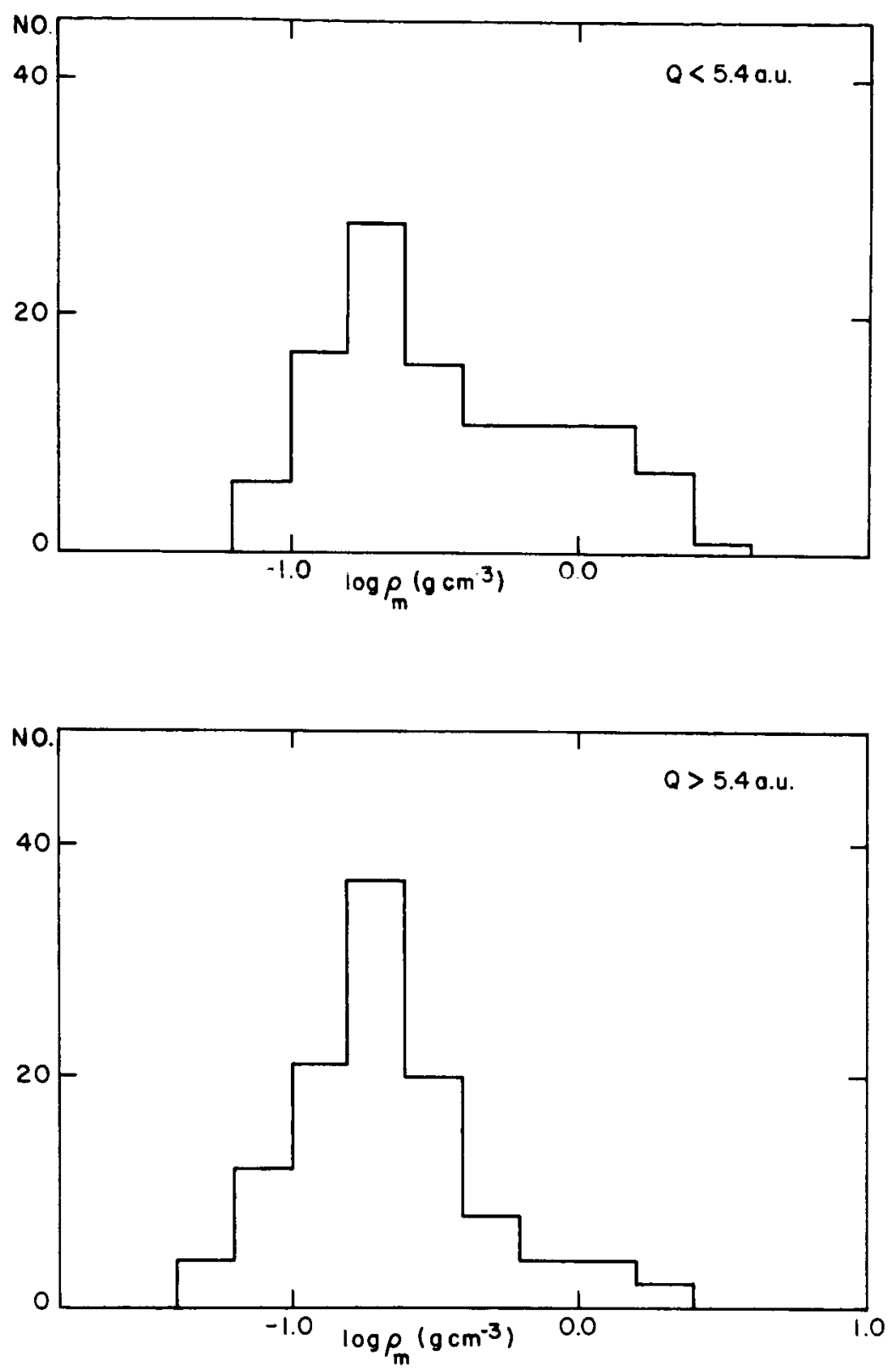

Figure 8. The density distribution for two groups of sporadic SuperSchmidt meteors, respectively, in short-and long-period orbits. 

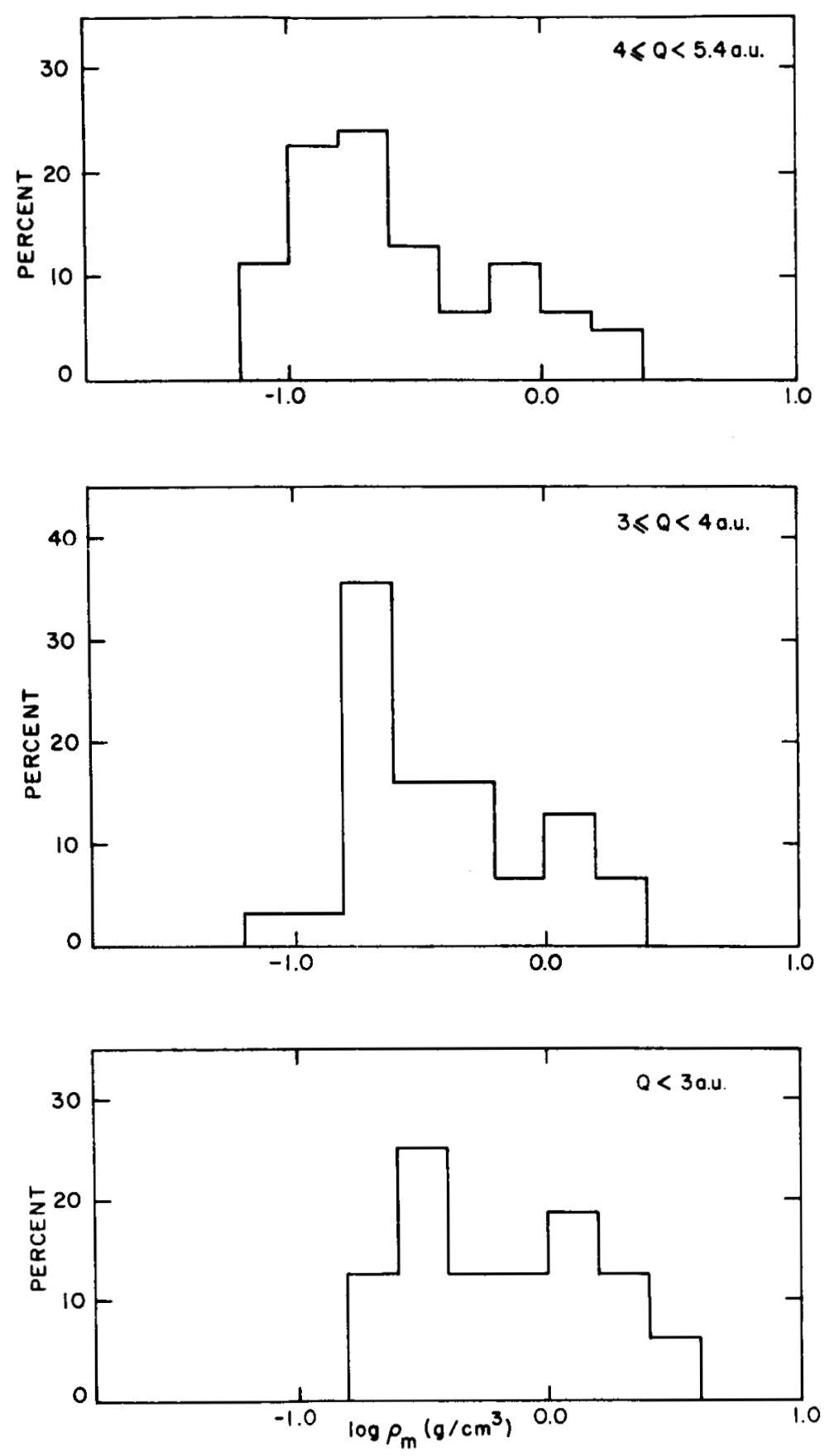

Figure 9. The density distribution for three subgroups of short-period sporadic meteors. 
of the mean density on $Q$ (Figure 7 ) is due only in part to the varying importance of the higher density group.

Among the 108 meteors with an aphelion distance smaller than $5.4 \mathrm{a}$. u., about 30 meteors are in the high-density group. The true consistence of this high-density group cannot be estimated simply by these figures. In fact, this sample of meteors is far from being random. In selecting the meteors to be reduced, Jacchia used the basic criterion that they should yield excellent decelerations, a fact that strongly favored the inclusion of long-trail meteors, and thus enhanced the probability of inclusion of high-density meteors (sec Jacchia, Verniani, and Briggs, 1965). It is nevertheless important to have established the probable existence of a small percentage of meteors in very short orbits having much higher densities than the great majority of all cometary meteors.

Among the original sample of 413 meteors, 123 belonged to wellestablished showers. Nineteen of these were disregarded for the following reasons: six had abrupt beginnings; three were flare-like; three did not allow the computation of $x$; two did not allow the computation of $\sigma$; four had too anomalous values of $\chi$; and finally, meteor no. 5180 , a member of the Northern Taurids, had physical characteristics drastically different from those of the meteors of the shower itself. Table 9 gives the results about the average density of each individual shower. For most of them, although the data are very scanty, the average density is near the average density for sporadic meteors. The most important exception, as is already well known (Jacchia, 1952; Verniani, 1964a; Jacchia, Verniani, and Briggs, 1965) a re the Geminids. On the average, they are about four times more dense than the sporadic meteors. At the other extreme, the Draconids have an extremely small density. In Figure 10, we have plotted the density distributions for Geminids, Southern Taurids and a Capricornids, i.e., the only showers with less scanty data. While the latter shows a distribution 


\section{Table 9. Average densities for Super-Schmidt shower meteors.}

\begin{tabular}{|c|c|c|c|c|c|}
\hline Showers: & $\begin{array}{c}\text { Mean } \log \rho_{\mathrm{m}} \pm \mathrm{s} . \mathrm{d} \\
\left.(\mathrm{g} \mathrm{cm})^{-3}\right)\end{array}$ & $\left.{ }_{(\mathrm{g} \mathrm{cm}}^{\rho_{\mathrm{m}}} \mathrm{cm}^{-3}\right)$ & $\begin{array}{l}\text { Median } \rho_{\mathrm{m}} \\
\qquad\left(\mathrm{g} \mathrm{cm}^{-3}\right)\end{array}$ & $\begin{array}{c}\text { Mean } Q \pm \text { s. d. } \\
\text { (a. u. ) }\end{array}$ & No. Obs. \\
\hline Geminids & $0.03 \pm 0.09$ & 1.06 & 1.14 & $2.6 \pm 0.0$ & 20 \\
\hline Southern Taurids & $-0.56 \pm 0.04$ & 0.28 & 0.25 & $3.4 \pm 0.2$ & 18 \\
\hline a Capricornids & $-0.85 \pm 0.07$ & 0.14 & 0.16 & $5.5 \pm 0.7$ & 12 \\
\hline Quadrantids & $-0.70 \pm 0.14$ & 0.20 & 0.17 & $5.0 \pm 0.2$ & 9 \\
\hline Perseids & $-0.54 \pm 0.07$ & 0.29 & 0.32 & $59+11$ & 8 \\
\hline$\delta$ Aquarids & $-0.57 \pm 0.07$ & 0.27 & 0.27 & $5.3 \pm 0.3$ & 7 \\
\hline Southern $v$ Aquarids & $-0.52 \pm 0.10$ & 0.30 & 0.32 & $4.7 \pm 0.6$ & 5 \\
\hline Orionids & $-0.60 \pm 0.08$ & 0.25 & 0.23 & $66 \pm 19$ & 4 \\
\hline$\kappa$ Cygnids & $-0.78 \pm 0.08$ & 0.17 & 0.17 & $5.3 \pm 0.4$ & 4 \\
\hline Northern Taurids & $-0.58 \pm 0.04$ & 0.26 & 0.27 & $4.7 \pm 0.7$ & 4 \\
\hline$\sigma$ Hydrids & $-0.40 \pm 0.18$ & 0.40 & 0.58 & $56 \pm 31$ & 3 \\
\hline Lyrids & $-0.41 \pm 0.13$ & 0.39 & 0.30 & $51 \pm 19$ & 3 \\
\hline Northern $v$ Aquarids & $-0.20 \pm 0.45$ & 0.63 & 0.63 & $3.6 \pm 0.6$ & 2 \\
\hline Draconids & - & $<0.01$ & - & $5.6 \pm 0.1$ & 2 \\
\hline Virginids & -0.13 & $\sim 0.7$ & - & 4.4 & 1 \\
\hline Le onids & -0.21 & $\sim 0.6$ & - & 24 & 1 \\
\hline$\eta$ Aquarids & -0.25 & $\sim 0.6$ & - & 26 & 1 \\
\hline
\end{tabular}

Note: In the second column, $\rho_{m}$ is the weighted logarithmic mean. 

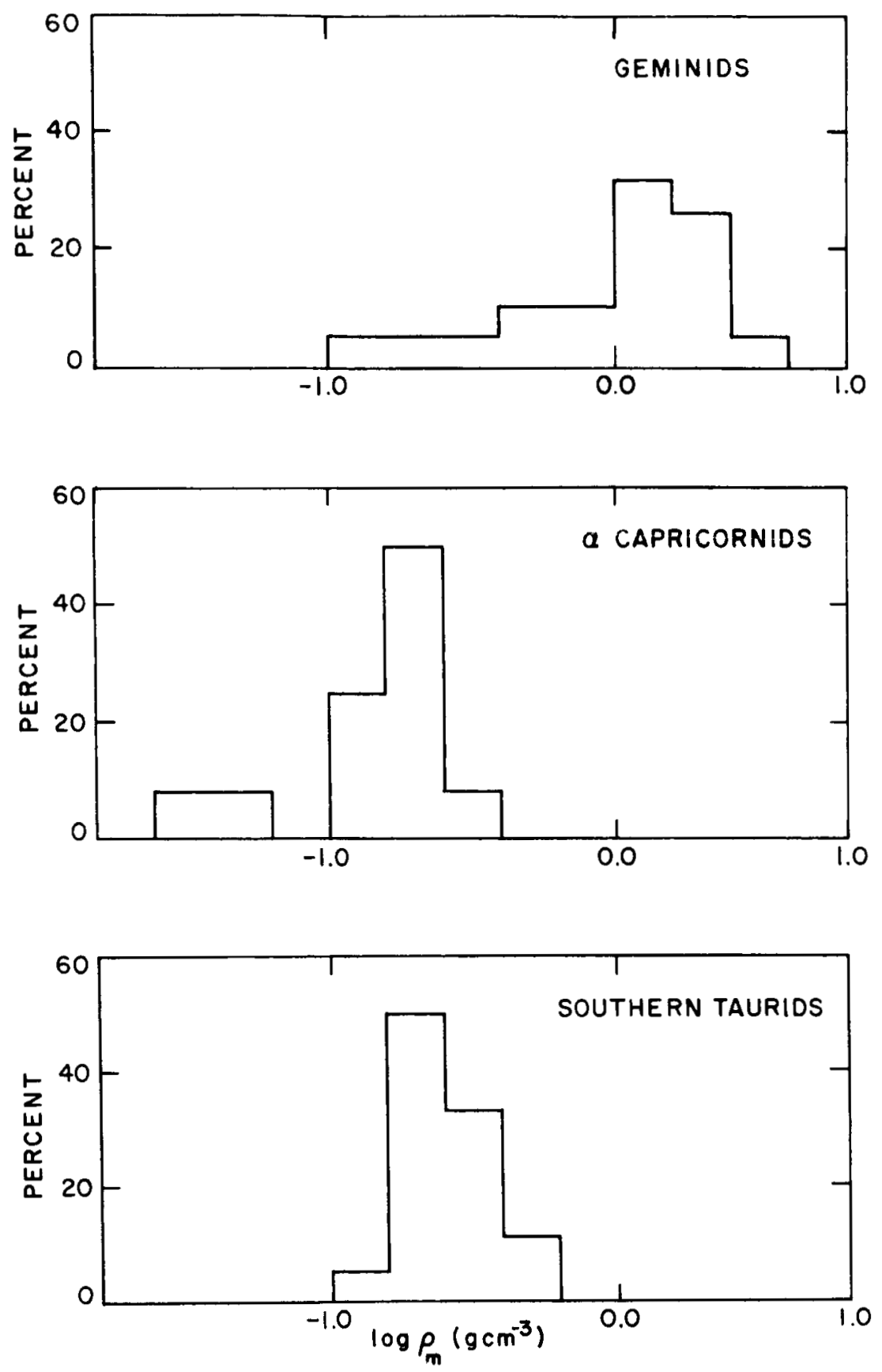

Figure 10. The density distribution for three meteor showers: the Geminids, a Capricornids, and Southern Taurids. 
more or less similar to that of long-period sporadic meteors, the Geminids have a distribution strongly resembling that of the highdensity group of meteors in very short orbits.

A summary of the results concerning shower and sporadic meteors is given in Table 10. On the average, there is no difference between sporadic and shower meteors. The mean density for all sporadic meteors $\left(0.28 \mathrm{~g} \mathrm{~cm}^{-3}\right)$ compares very well with the density found by the author (Verniani, 1964b) for 284 faint Super-Schmidt meteors, $0.30 \mathrm{~g} \mathrm{~cm}^{-3}$, when we correct for the shape factor, which was then assumed to be 1.21 instead of 1.5. Preliminary results for ineteors detected by radar methods, having an average mass $10^{4}$ times smaller than those discussed in this paper, indicate a somewhat larger value for the density, of the order of $0.8 \mathrm{~g} \mathrm{~cm}^{-3}$ (Verniani and Hawkins, 1965; Verniani, 1966). 
Table 10. Summary of the densities of 322 Super-Schmidt meteors.

$\begin{array}{lcccc} & \text { Mean log } \rho_{\mathrm{m}} \pm \mathrm{s.d.} & \rho_{\mathrm{m}} & \text { Median } \rho_{\mathrm{m}} \\ & \left(\mathrm{g} \mathrm{cm}^{-3}\right) & \left(\mathrm{g} \mathrm{cm}^{-3}\right) & \left(\mathrm{g} \mathrm{cm}^{-3}\right) & \text { No. Obs. } \\ \text { All meteors } & -0.533 \pm 0.021 & 0.29 & 0.25 & 322 \\ \text { Shower meteors } & -0.474 \pm 0.037 & 0.34 & 0.27 & 102 \\ \text { Shower meteors, Geminids excluded } & -0.590 \pm 0.029 & 0.26 & 0.24 & 82 \\ \text { Sporadic metenrs } & -0.558 \pm 0.025 & 0.28 & 0.23 & 220 \\ \text { Sporadic meteros with Q }<5.4 \text { a.u. } & -0.458 \pm 0.039 & 0.35 & 0.26 & 108 \\ \text { Sporadic meteors with Q }>5.4 \text { a.u. } & -0.671 \pm 0.029 & 0.21 & 0.20 & 112 \\ \text { Sporadic low-density meteors } & -0.673 \pm 0.019 & 0.21 & 0.21 & 189 \\ \text { Sporadic high-density meteors } & 0.140 \pm 0.024 & 1.38 & 1.46 & 31\end{array}$

Note: In the second column, $\rho_{m}$ is the weighted logarithmic mean. 


\section{REFERENCES}

CEPLECHA, Zd, , RAJCHL, J., and SEHNAL, L.

1959. Complete data on bright meteor 15761. Bull. Astron. Inst. Czechoslovakia, vol. 10, pp. 204-212。

COOK, A. F.

1966. Personal communication.

COOK, A. F., JACCHIA, L. G., and MCCROSKY, R. E。

1963. Luminous efficiency of iron and stone asteroidal meteors.

Smitlisunian Contr. Astrophys., vol. 7, pp. 209-220.

HALLIDAY, I.

1960. The spectrum of an asteroidal meteor fragment. Astrophys.

Journ., vol. 132, pp. $482-485$.

HERLOFSON, N.

1948. The theory of meteor ionization. Rep. Prog. Phys., vol.

$\mathrm{XI}$, pp. $444-454$.

JACCHIA, L. G.

1949. Photographic rineteor phenomena and theory. Harvard

College Obs. and Center Anal. M.I. T., Tech. Rep. No. 3, Harvard Reprint Series II, No. 31, 36 pp.

1952. A comparative analysis of atmospheric densities from meteor decelerations observed in Massachusetts and New Mexico. Harvard College Obs. and Numer. Anal. Lab. of M.I. T., Tech. Rep. No. 10, Harvard Reprint Series II, No. $44,37 \mathrm{pp}$.

1955. The physical theory of meteors. VIII. Fragmentation as cause of the faint-meteor anaomaly. Astrophys. Journ., vol. 121, pp. 521-527.

JACCHIA, L. G., VERNIANI, F., and BRIGGS, R. E.

1965. An analysis of the atmospheric trajectories of 413 precisely reduced photographic meteors. Smithsonian Astrophys. Obs.Spec. Rep., No. 175, 309 pp. 
MCCROSKY , R. E.

1966. Personal communication.

MCCROSKY, R. E., and SOBERMAN, R. K.

1963. Results from an artificial iron meteoroid at $10 \mathrm{~km} / \mathrm{sec}$.

Smithsonian Contr. Astrophys., vol. 7, pp. 199-208.

MINZNER, R. A., CHAMPION, K. S. W., and POND, H. L.

1959. The ARDC model atmosphere, 1959. Air Force Surveys in Geophys., No. 115, Geophys. Res. Dir., AFCRC-TR59-267, $137 \mathrm{pp}$.

U.S. STANDARD ATMOSPHERE 1962

1962. Prepared under NASA, USAF, and USWB, U.S. Gov't.

Printing Office, Washington, D. C. , 278 pp.

VERNIANI, F.

1964a. On the luminous efficiency of meteors. Smithsonian

Astrophys. Obs. Spec. Rep. No. 145, 62 pp. (Also

published in Smithsonian Contr. Astrophys., vol. 8,

pp. 141-172, 1965).

$1964 \mathrm{~b}$. On the density of meteoroids. II. The density of faint photographic meteoroids. Nuovo Cimento, vol. 33, no. 4, pp. 1173-1184.

1966. Physical characteristics of 320 faint radio meteors. Journ. Geophys. Res., vol. 71, pp. 2749-2761.

VERNIANI, F., and HAWKINS, G。S.

1964. On the ionizing efficiency of meteors. Astrophys. Journ., vol. 140, pp. 1590-1600.

1965. Masses, magnitudes, and densities of 320 radio meteors. Harvard Radio Meteor Project, Res. Rep. No. 12, 35 pp.

WHIPPLE, F. L.

1963. On meteoroids and penetration. Journ. Geophys. Res., vol. 68, no. 17, pp. 4929-4939. 
WOLFE, H. C.

1962. On uniformity of international usage. General introduction to Symbols, Units, and Nomenclature in Physics, prepared by the Commission on Symbols, Units and Nomenclature, Int'l. Union of Pure and Applied Physics, Physics Today, vol. 15, pp. 19-30.

WOOD, J. A.

1963. Physics and chemistry of meteorites. In The Moon, Meteorites, and Comets. The Solar System, vol. IV, ed. by B. M. Middlehurst and G. P. Kuiper, Univ. of Chicago Press, Chicago, pp. 337-401. 


\section{NOTICE}

This series of Special Reports was instituted under the supervision of Dr. F. L. Whipple, Director of the Astrophysical Observatory of the Smithsonian Institution, shortly after the launching of the first artificial earth satellite on October 4, 1957. Contributions come from the Staff of the Observatory.

First is sued to ensure the immediate diss emination of data for satellite tracking, the reports have continued to provide a rapid distribution of catalogs of satellite observations, orbital information, and preliminary results of data analyses prior to formal publication in the appropriate journals. The Reports are also used extensively for the rapid publication of preliminary or special results in other fields of astrophysics.

The Reports are regularly distributed to all institutions participating in the U.S. space research program and to individual scientists who request them from the Publications Division, Distribution Section, Smithsonian Astrophysical Observatory, Cambridge, Massachusetts 02138. 